\title{
The Number Process as Low Density Limit of Hamiltonian Models
}

\author{
L. Accardi and Y. G. Lu *
}

Centro Matematico V. Volterra and Dipartimento di Matematica, Università di Roma II, Italy

Received June 22, 1989; in revised form February 13, 1991

\begin{abstract}
We study a nonrelativistic quantum system coupled, via a quadratic interaction [cf. formula (1.10) below], to a free Boson gas in the Fock state. We prove that, in the low density limit $\left(z^{2}=\right.$ fugacity $\left.\rightarrow 0\right)$, the matrix elements of the wave operator of the system at time $t / z^{2}$ in the collective coherent vectors converge to the matrix elements, in suitable coherent vectors of the quantum Brownian motion process, of a unitary Markovian cocycle satisfying a quantum stochastic differential equation driven by some pure number process (i.e. no quantum diffusion part and only the quantum analogue of the purely discontinuous, or jump, processes). This proves that the number (or quantum Poisson) processes, introduced by Hudson and Parthasarathy and studied by Frigerio and Maassen, arise effectively as conjectured by the latter two authors as low density limits of Hamiltonian models.
\end{abstract}

\section{Introduction}

The study of the weak coupling (van Hove) or low density limit of quantum Hamiltonian systems is framed within the wider program of understanding the origins of irreversible behaviours in quantum phenomena. In this study three stages of developments can be recognized: (i) a first one, in which the driving scales of magnitude are individuated and the irreversible equations are deduced on a phenomenological basis (van Hove scaling, Pauli master equation, WignerWeiskopf approximation); (ii) a second one, in which the various types of master equations are deduced from Hamiltonian models; (iii) a third one, in which, starting from the same types of models as in (ii), one tries to derive not only the master equation, but the full quantum Langevin equation.

The master equation is an ordinary differential equation, describing the reduced evolution of the system, obtained from the full Heisenberg evolution by taking the

* On leave of absence from Beijing Normal University 
partial expectation with respect to the vacuum state of the reservoirs degrees of freedom. The quantum Langevin equation is a quantum stochastic differential equation driven by some quantum noise (creation, annihilation, number noises). It describes the full evolution of the coupled (system + reservoir) system. Taking the partial expectation, with respect to the vacuum, of the quantum Langevin equation, one obtains the master equation.

From the physical point of view, the passage from step (ii) to step (iii) means that one wants to study the limiting behaviour not only of the system, but also of the reservoir degrees of freedom. Since the reservoirs are usually modeled as quantum fields or Bose or Fermi gases, this means that the basic issue is to understand in what sense the quantum Brownian motion and the number process are approximations of usual quantum fields.

From the mathematical point of view, the basic difficulty in the passage from level (ii) to level (iii) is that, at level (ii), the limit of the reduced evolution is considered with respect to some standard operator topology (usually the weak, but sometimes also the trace-norm topology, is used). Nothing similar could be hoped for level (iii) because even in the analogous classical case one can only prove convergence in law of the corresponding probabilities.

In a series of papers [3-7] we have solved the problem (iii) in the case of the weak coupling limit of various kinds of nonrelativistic quantum systems coupled, via linear or quadratic interactions, to a free Bose or Fermi gas in some quasi-free state. We have proved, for all these systems, the convergence in the sense of matrix elements in some appropriate collective vectors of the wave operator at time $t / \lambda^{2}(\lambda$ being the coupling constant) to a unitary Markovian cocycle satisfying a quantum stochastic differential equation of diffusion type (i.e. driven only by the creation and annihilation martingales). We have also proved convergence of the Heisenberg evolution of an observable of the system to the solution of a quantum Langevin equation. A qualitatively new feature of our approach is that the term collective does not refer to the special degrees of freedom, but to time [cf. (1.17a)]. This choice is not arbitrary, but suggested by $1^{\text {st }}$ order perturbation theory.

In the weak coupling limit, only the quantum Brownian motions arise (and these quantum noises were well known in quantum optics), but we know from Hudson and Parthasarathy [17] and Frigerio and Maassen [14], that there is another quantum noise, quite natural and important from the mathematical point of view: the quantum Poisson (or number) processes. This is defined as follows:

Let $\Gamma(H)$ denote the Fock space over a Hilbert space. $T$ is a self-adjoint operator on $H$ and $\Phi(f)$ the normalized coherent vector in $\Gamma(H)$ with test function $f$. The generator $N(T)$ of the one-parameter unitary group $\Gamma\left(e^{i \lambda T}\right)$ characterized by the property

$$
\Gamma\left(e^{i \lambda T}\right) \Phi(f)=\Phi\left(e^{i \lambda T} f\right) ; \quad \lambda \in \mathbf{R}
$$

is called the number operator associated to $T$ and is characterized by the property

$$
\langle\Phi(f), N(T) \Phi(g)\rangle=\langle f, T g\rangle \cdot\langle\Phi(f), \Phi(g)\rangle .
$$

The definition of $N(T)$ is extended by complex linearity to any bounded operator $T$ on $H$. If $H$ is of the form $L^{2}(\mathbf{R}, d t ; \mathscr{K})\left(\cong L^{2}(\mathbf{R}) \otimes \mathscr{K}\right)$ for some Hilbert space $\mathscr{K}$, then for any bounded operator $T$ on $\mathscr{K}$ and for any $t \geqq 0$, one can define

$$
N_{t}(T):=N\left(\chi_{[0, t]} \otimes T\right) .
$$

The family $\left\{N_{t}(T) ; t \geqq 0\right\}$ is called number process with strength $T$. Its connection with the classical Poisson process is explained in [14]. Contrary to the quantum 
Brownian motions, the number processes did not show up in the physical literature, even at a phenomenological level. Frigerio and Maassen [14] and later Frigerio and Alicki [9] conjectured, on the basis of plausible physical considerations, that the number processes should arise naturally in the low density limit, in analogy to what happens for quantum Brownian motion in the weak coupling limit. More precisely they conjectured that in the low density limit of the Heisenberg evolution considered by Dümcke [11] one should obtain a quantum stochastic equation driven by the quantum Poisson noise in the sense of [14] and that this equation determines a dilation in the sense of Kümmerer [20] of the semigroup obtained by Dümcke in [11].

In order to realize this program the first step is to understand the mechanism through which a quantum stochastic equation, driven by pure number processes can arise as a limit of usual Hamiltonian equations.

This step has, of course, no analogue in the previous literature, because in the reduced evolution approach the quantum noises were swept away by the vacuum expectation and only the semigroup survived. On the contrary here we are mainly interested in the mechanism giving rise to the quantum noise and the main result of the present paper is the discovery of this mechanism [cf. Theorem (6.2) below]. In particular, our result explains why the physical effects of the number process were discovered almost 30 years after those of the quantum Brownian motion: the point is that the coefficients of the quantum noises in the stochastic differential equation depend at most quadratically on the coefficients of the field operators in the original Hamiltonian model. Thus the quantum Brownian motion is essentially a second order effect. On the contrary, our main result shows that the coefficients of the number process receive contributions from the terms of all orders in the perturbation series. Therefore, to isolate the physical effects of the number processes, a much more detailed analysis of the perturbation series is required.

Our main strategy, of which the present paper constitutes the first and most important step, can be described as follows: we start from a quadratic interaction of the form (1.9), defined on some finite temperature representation of the CCR with a given fugacity $z^{2}$ [cf.(1.3),(1.4) below]. Following Palmer [18] we realize the representation space as the tensor product of a Fock and an anti-Fock representation. In this realization, the interaction (1.9) splits into 3 pieces: one of order $z^{2}$, that we expect to vanish in the limit; one of order $z$, which has the structure of a weak coupling limit term, and therefore controllable with the techniques developed in [3-7]; finally, one of order zero, which lives only on the Fock space. The order zero term is the only qualitatively new feature with respect to the weak coupling case, hence we expect that, if the number process arises at all in the limit, it should come from this term. This is plausible from the physical point of view because also in the classical case the Poisson distribution arises from interactions which are of finite intensity but very rare (low density). This suggested the program of concentrating our attention to this piece of the interaction, in the hope that, by doing so, we could isolate the basic contribution to the appearance of the number process by means of an adaptation of the techniques developed in order to deal with the weak coupling limit. It turns out that this is indeed the case.

\section{Notations}

Following the pattern of [3], we formulate the problem for a general quasi-free state and we prove the convergence of the kinematical process of the collective 
coherent vectors to quantum Brownian motion in the general case. Starting from Sect. 3 we restrict our attention to the Fock case.

Let $H_{0}$ denote the system Hilbert space and $H_{1}$ the one particle reservoir Hilbert space. Let

$$
W\left(H_{1}\right):=\left\{W(f): f \in H_{1}\right\}
$$

be the Weyl $C^{*}$-algebra on $H_{1}$; let $H$ be a self-adjoint operator bounded below on $H_{1}$ and $z, \beta$ positive real numbers interpreted respectively as density of the reservoir particles and inverse temperature. Define

$$
Q_{z}:=\left(1+z^{2} e^{-\beta H}\right) \cdot\left(1-z^{2} e^{-\beta H}\right)^{-1}
$$

and suppose that, for each $z$ in an interval $[0, Z], Q_{z}$ is a self-adjoint operator on a domain $D$, independent on $z$. In the fermion case (1.2) is replaced by

$$
Q_{z}:=\left(1-z^{2} e^{-\beta H}\right) \cdot\left(1+z^{2} e^{-\beta H}\right)^{-1}
$$

which is a bounded operator for each $z$. Denote $\varphi_{Q_{z}}$ the mean zero gauge invariant quasi-free state on $W\left(H_{1}\right)$ with covariance operator $Q_{z}$, i.e.

$$
\varphi_{Q_{z}}(W(f))=\exp \left(-\frac{1}{2}\left\langle f, Q_{z} f\right\rangle\right), \quad \forall f \in H_{1}
$$

and let $\left\{\mathscr{H}_{Q_{z}}, \pi_{Q_{z}}, \Phi_{Q_{z}}\right\}$ be the GNS-triple of $\left\{W\left(H_{1}\right), \varphi_{Q_{z}}\right\}$, so that

$$
\left\langle\Phi_{Q_{z}}, \pi_{Q_{z}}(W(f)) \Phi_{Q_{z}}\right\rangle=\varphi_{Q_{z}}(W(f)) .
$$

We shall write $W_{Q_{z}}$ for $\pi_{Q_{z}} \circ W$. The Fock representation corresponds to the case $Q_{z}=1$, i.e. $\beta=\infty$. In this case the GNS representation will be simply denoted $\{\mathscr{H}, \pi, \Phi\}$. Let $S_{t}$ be a unitary group on $B\left(H_{1}\right)$ (the one particle free evolution of the reservoir) and suppose that

$$
S_{t} \cdot Q_{z}=Q_{z} \cdot S_{t}, \quad \forall t \geqq 0,
$$

where the equality is meant on $D$. This implies that the second quantization of $S_{t}$, denoted $\Gamma\left(S_{t}\right)$, leaves $\varphi_{Q_{z}}$ invariant hence it is implemented, in the GNS representation, by a unitary 1-parameter group $V_{t}^{(z)}$ whose generator $H^{(z)}=: H_{R}$ is called the free Hamiltonian of the reservoir. As in [3] we assume that there exists a non-zero subspace $K$ of $H_{1}$ (in all the examples it is a dense subspace) such that

$$
\int_{\mathbf{R}}\left|\left\langle f, S_{t} g\right\rangle\right| d t<\infty, \quad \forall f, g \in K .
$$

Moreover, we suppose that $Q_{z} K \cong K$. For example, for the free Bose gas,

$$
H_{R}=d \Gamma(-\Delta),
$$

where $\Delta$ is the Laplacian on $L^{2}\left(\mathbf{R}^{d}\right)$ with $d \geqq 3$. Let be given a self-adjoint operator $H_{S}$ on the system space $H_{0}$, called the system Hamiltonian. The total free Hamiltonian is defined to be

$$
H^{(0)}:=H_{S} \otimes 1+1 \otimes H_{R} .
$$

We define the interaction Hamiltonian $V$ as in [11] and [18] i.e., we fix two functions $g_{1}, g_{0} \in K$ and define

$$
\begin{aligned}
& V: i\left(D \otimes A^{+}\left(g_{0}\right) \cdot A\left(g_{1}\right)-D^{+} \otimes A^{+}\left(g_{1}\right) \cdot A\left(g_{0}\right)\right) \\
& \quad=i \sum_{\varepsilon \in\{0,1\}} D_{\varepsilon} \otimes A^{+}\left(g_{\varepsilon}\right) \cdot A\left(g_{1-\varepsilon}\right)
\end{aligned}
$$


with the notations

$$
D_{0}=D, \quad D_{1}=-D^{+},
$$

and where $D$ is a bounded operator on $H_{0}$ satisfying

$$
\exp \left(i t H_{S}\right) \cdot D \cdot \exp \left(-i t H_{S}\right)=D \text {. }
$$

Moreover, we assume that $g_{0}$ and $g_{1}$ have disjoint energy spectra, i.e.

$$
\left\langle g_{0}, S_{t} g_{1}\right\rangle=0, \quad \forall t \in \mathbf{R} \text {. }
$$

More general interactions will be discussed in subsequent papers.

The condition (1.12) is natural because of the condition (2.5) of [13] and has already been used in the literature on the weak coupling limit (cf. [10, Sect. 3]). With the condition (1.12), the condition (1.11) is also natural since a typical example for $D$ in quantum optics is $D=|0\rangle\langle 1|$, where $|1\rangle,|0\rangle$ are eigenvectors of the system Hamiltonian $H_{S}$ (rotating wave approximation). This corresponds to $\left[H_{S}, D\right]=\left(\omega_{1}-\omega_{0}\right) D\left(\omega_{1}, \omega_{0}\right.$ are the eigenvalues). The condition (1.11) corresponds to taking $\omega_{1}=\omega_{0}$, but the choice $\omega_{1} \neq \omega_{0}$ results only in a trivial shift in the one particle reservoir Hamiltonian (cf. Sect. 5 in [21] for the detail).

With these notations, the total Hamiltonian is

$$
H_{\text {total }}:=H_{S} \otimes 1+1 \otimes H_{R}+V
$$

and the wave operator at time $t$ is defined by

$$
U_{t}:=\exp \left(i t H^{(0)}\right) \cdot \exp \left(-i t H_{\text {total }}\right) .
$$

Therefore, we have the formal identity

where,

$$
\frac{d}{d t} U_{t}=\frac{1}{i} V(t) U_{t} ; \quad U(0)=\mathbf{1}
$$

$$
V(t):=\exp \left(i t H^{(0)}\right) V \exp \left(-i t H^{(0)}\right)=\mathrm{i} \sum_{\varepsilon \in\{0,1\}} D_{\varepsilon} \otimes A^{+}\left(S_{t} g_{\varepsilon}\right) A\left(S_{t} g_{1-\varepsilon}\right) .
$$

Moreover, the solution of $(1.15)$ is given by the iterated series

$$
U_{t}=\sum_{n=0}^{\infty}(-i)^{n} \int_{0}^{t} d t_{1} \ldots \int_{0}^{t_{n-1}} d t_{n} V\left(t_{1}\right) \ldots V\left(t_{n}\right)
$$

which is, under the condition stated in Sect. 5, weakly convergent on the domain of the vectors of the form

$$
u \otimes \Phi_{Q_{z}}\left(z \int_{S / z^{2}}^{T / z^{2}} S_{u} f d u\right),
$$

where $u \in H_{0}, f \in K, S, T \in \mathbf{R}$ and

$$
\Phi_{Q_{z}}\left(z \int_{S / z^{2}}^{T / z^{2}} S_{u} f d u\right):=W_{Q_{z}}\left(z \int_{S / z^{2}}^{T / z^{2}} S_{u} f d u\right) \Phi_{Q_{z}}
$$

is a collective coherent vector in the sense of [3] and the identitiy (1.15) holds rigorously in the topology on this domain. From Lemma (3.2) of [3], we know that the assumption (1.7) implies that the sesquilinear form $(\cdot \mid \cdot): K \times K \rightarrow \mathscr{C}$ defined by

$$
(f \mid g):=\int_{\mathbf{R}}\left\langle f, S_{t} g\right\rangle d t, \quad f, g \in K,
$$


defines a pre-scalar product on $K$. We denote $\{K,(\cdot \mid \cdot)\}$, or simply $K$, the completion of the quotient of $K$ by the zero $(\cdot \mid \cdot)$-norm elements. In the following we shall also use the quantity

$$
(f \mid g)_{-}:=\int_{-\infty}^{0}\left\langle f, S_{t} g\right\rangle d t, \quad f, g \in K .
$$

In [11 and 18] it was proved that, under some conditions, the limit

$$
\lim _{z \rightarrow 0} \varphi \otimes \varphi_{Q_{z}}\left(U_{t / z^{2}}(X \otimes 1) U_{t / z^{2}}^{+}\right)
$$

exists in a small time interval and is equal to

$$
\varphi\left(T_{t}(X)\right),
$$

where $\left\{T_{t}\right\}_{t \geq 0}$ is a quantum Markovian semigroup. The analogy with the new techniques, developed in [3], for the weak coupling limit, suggests to consider the limit, as $z \rightarrow 0$ of expressions of the form

$$
\langle u,(\cdot) v\rangle \otimes \varphi_{Q_{z}}\left(1 \otimes W\left(f_{z}\right) U_{t / z^{2}}(X \otimes 1) U_{t / z^{2}}^{+} \mathbf{1} \otimes W\left(f_{z}^{\prime}\right)\right),
$$

where

$$
f_{z}=z \int_{S / z^{2}}^{T / z^{2}} S_{u} f d u, \quad f \in K .
$$

In the control of the limit, as $z \rightarrow 0$ of the expressions (1.21), the basic difference between the low density and the weak coupling case is that in the low density case the wave operator $U_{t / z^{2}}$ depends on $z$ (the density) only in the rescaling of the time $\left(t \mapsto t / z^{2}\right)$, while in the weak coupling case the wave operator $U_{t / z^{2}}^{(z)}$ depends on $z$ (the coupling constant) in its very structure. On the other hand, the reservoir state is independent on $z$ in the weak coupling limit, but dependent on it in the low density limit.

In analogy with the strategy of [3], the first step in our investigation will be to control the limit of expressions of the form

$$
\lim _{z \rightarrow 0}\left\langle u \otimes W\left(z \int_{S / z^{2}}^{T / z^{2}} S_{u} f d u\right) \Phi, U_{t / z^{2}} \cdot v \otimes W\left(z \int_{S^{\prime} / z^{2}}^{T^{\prime} / z^{2}} S_{u} f^{\prime} d u\right) \Phi\right\rangle
$$

in the Fock case. This will be done in the present paper. Exactly as in the weak coupling limit case, the estimates needed to solve this problem will allow, with minor modifications, to control more general situations (cf. [4]). In order to formulate our result, let us recall from [3] the definition of Quantum Brownian Motion:

Definition (1.1). Let $\mathscr{K}$ be a Hilbert space, $T$ an interval in $\mathbf{R}$. Let $Q \geqq 1$ be a selfadjoint operator on $\mathscr{K}$ and let

$$
\left\{\mathscr{H}_{Q}, \pi_{Q}, \Phi_{Q}\right\}
$$

denote the GNS representation of the CCR over $L^{2}(T, d t ; \mathscr{K})$ with respect to the quasi-free state $\varphi_{Q}$ on $W\left(L^{2}(T, d t ; \mathscr{K})\right)$ characterized by

$$
\varphi_{Q}(W(\xi))=e^{-1 / 2\langle\xi, 1 \otimes Q \xi\rangle} ; \quad \xi \in L^{2}(T, d t ; \mathscr{K}) .
$$

The stochastic process

$$
\left\{\Gamma\left(L^{2}(T, d t ; \mathscr{K})\right), A\left(\chi_{(s, t]} \otimes f\right), A^{+}\left(\chi_{(s, t]} \otimes f\right) ;(s, t] \leqq T, f \in \mathscr{K}\right\},
$$


where $A(\cdot), A^{+}(\cdot)$ denote respectively the annihilation and creation fields in the representation (1.27), is called the $Q$-Quantum Brownian Motion on $L^{2}(T, d t ; \mathscr{K})$.

Our main result in this paper is to prove that the limit (1.23) exists and is equal to

$$
\left\langle u \otimes W\left(\chi_{[S, T]} \otimes f\right) \Psi, U(t) \cdot v \otimes W\left(\chi_{\left[S^{\prime}, T^{\prime}\right]} \otimes f^{\prime}\right) \Psi\right\rangle,
$$

where $\{\mathscr{H}, W, \Psi\}$ with $\mathscr{H}:=\Gamma\left(L^{2}(T, d t ; K)\right)$ is the Fock-Brownian motion on $L^{2}(\mathbf{R}, d t ; K)$ and $U(t)$ satisfies a quantum stochastic differential equation driven by purely discontinuous noises in the sense of $[14,17]$, whose form is given by $(6.1)$.

\section{Convergence of the Collective Process}

In this section we show that, at a purely kinematical level, i.e. with $t=0$ in (1.23), the low density limit coincides with the weak coupling limit and the limiting process is the Fock-Brownian motion on $L^{2}(\mathbf{R}, d t ; K)$, where $K$ is equipped with the scalar product (1.18). has

First, recall from $\left[3\right.$, Lemma (3.2)] that for each $f, f^{\prime} \in K$ and $S, S^{\prime}, T, T^{\prime} \in \mathbf{R}$, one

$$
\begin{aligned}
\lim _{z \rightarrow 0} & \left\langle z \int_{S / z^{2}}^{T / z^{2}} S_{u} f d u, z \int_{S^{\prime} / z^{2}}^{T^{\prime} / z^{2}} S_{u} f^{\prime} d u\right\rangle \\
& =\left\langle\chi_{[S, T]}, \chi_{\left[S^{\prime}, T^{\prime}\right]}\right\rangle_{L^{2}(\mathbf{R})} \cdot \iint_{\mathbf{R}}\left\langle f, S_{t} f^{\prime}\right\rangle d t \\
& =\left\langle\chi_{[S, T]} \otimes f, \chi_{\left[S^{\prime}, T^{\prime}\right]} \otimes f^{\prime}\right\rangle_{L^{2}(\mathbf{R}, d t ; K)} .
\end{aligned}
$$

Lemma (2.1). For each $n \in \mathbf{N},\left\{f_{k}\right\}_{k=1}^{n} \subset K,\left\{S_{k}, T_{k}\right\}_{k=1}^{n} \subset \mathbf{R},\left\{x_{k}\right\}_{k=1}^{n} \subset \mathbf{R}$,

$$
\begin{aligned}
& \lim _{z \rightarrow 0}\left\langle\Phi_{Q_{z}}, W\left(x_{1} z \int_{S_{1} / z^{2}}^{T_{1} / z^{2}} S_{u} f_{1} d u\right) \ldots W\left(x_{n} z \int_{S_{n} / z^{2}}^{T_{n} / z^{2}} S_{u} f_{n} d u\right) \Phi_{Q_{z}}\right\rangle \\
& \quad=\left\langle\Psi, W\left(x_{1} \chi_{\left[S_{1}, T_{1}\right]} \otimes f_{1}\right) \ldots W\left(x_{n} \chi_{\left[S_{n}, T_{n}\right]} \otimes f_{n}\right) \Psi\right\rangle
\end{aligned}
$$

and the convergence is uniform for $\left\{x_{k}\right\}_{k=1}^{n},\left\{S_{k}, T_{k}\right\}_{k=1}^{n}$ in a bounded set of $\mathbf{R}$, where, $\Psi$ is the vacuum of $\Gamma\left(L^{2}(\mathbf{E}, d t ; K)\right)$.

Proof. In the above notations one has

$$
\begin{aligned}
\left\langle\Phi_{Q_{z}}, W\left(\int_{S_{1} / z^{2}}^{T_{1} / z^{2}} S_{u} f_{1} d u\right) \ldots W\left(x_{n} z \int_{S_{n} / z^{2}}^{T_{n} / z^{2}} S_{u} f_{n} d u\right) \Phi_{Q_{z}}\right\rangle \\
=\exp \left(-\operatorname{Im} \sum_{1 \leqq j<k \leqq n} x_{j} x_{k} z^{2} \int_{S_{j} / z^{2}}^{T_{j} / z^{2}} \int_{S_{k} / z^{2}}^{T_{k} / z^{2}} d u d v\left\langle S_{u} f_{j}, S_{v} f_{k}\right\rangle\right) \\
\quad \times\left\langle\Phi_{Q_{z}}, W\left(\sum_{k=1}^{n} x_{k} z \int_{S_{k} / z^{2}}^{T_{k} / z^{2}} S_{u} f_{k} d u\right) \Phi_{Q_{z}}\right\rangle \\
=\exp \left(-\operatorname{Im} \sum_{1 \leqq j<k \leqq n} x_{j} x_{k} z^{2} \int_{S_{j} / z^{2}}^{T_{j} / z^{2}} \int_{S_{k} / z^{2}}^{T_{k} / z^{2}} d u d v\left\langle S_{u} f_{j}, S_{v} f_{k}\right\rangle\right) \\
\quad \times \exp \left(-\frac{1}{2}\left\langle\sum_{k=1}^{n} x_{k} z \int_{S_{k} / z^{2}}^{T_{k} / z^{2}} S_{u} f_{k} d u, \sum_{k=1}^{n} x_{k} z \int_{S_{k} / z^{2}}^{T_{k} / z^{2}} S_{u} Q_{z} f_{k} d u\right\rangle\right),
\end{aligned}
$$

and the only difference with the situation considered in Theorem (3.4) of [3] is the presence of the term $Q_{z}$ (instead of a $z$-independent $Q$ ). By definition, one has

$$
Q_{z}=\left(1+z^{2} e^{-\beta H}\right) \cdot\left(1-z^{2} e^{-\beta H}\right)^{-1}=1+2 \sum_{n=1}^{\infty} z^{2 n} e^{-\beta n H}
$$


and the convergence is in norm since $H$ is bounded below. So, by (2.1), for each $1 \leqq j$ $<k \leqq n$,

$$
\begin{aligned}
& z^{2}\left\langle\int_{S_{k} / z^{2}}^{T_{k} / z^{2}} S_{u} f_{k} d u, \int_{S_{j} / z^{2}}^{T_{j} / z^{2}} S_{u} Q_{z} f_{j} d u\right\rangle \\
& =z^{2}\left\langle\int_{S_{k} / z^{2}}^{T_{k} / z^{2}} S_{u} f_{k} d u, \int_{S_{j} / z^{2}}^{T_{j} / z^{2}} S_{u} f_{j} d u\right\rangle+z^{2} O(1) \rightarrow\left\langle\chi_{\left[S_{k}, T_{k}\right]} \otimes f_{k}, \chi_{\left[S_{j}, T_{j}\right]} \otimes f_{j}\right\rangle .
\end{aligned}
$$

Hence,

$$
\begin{aligned}
\lim _{z \rightarrow 0} & \left\langle\Phi_{Q_{z}}, W\left(x_{1} z \int_{S_{1 / z^{2}}}^{T_{1} / z^{2}} S_{u} f_{1} d u\right) \ldots W\left(x_{n} z \int_{S_{n} / z^{2}}^{T_{n} / z^{2}} S_{u} f_{n} d u\right) \Phi_{Q_{z}}\right\rangle \\
= & \exp \left(-\frac{1}{2} \sum_{1 \leqq j, k \leqq n} x_{k} x_{j}\left\langle\chi_{\left[S_{k}, T_{k}\right]} \otimes f_{k}, \chi_{\left[S_{j}, T_{j}\right]} \otimes f_{j}\right\rangle\right) \\
& \times \exp \left(-\operatorname{Im} \sum_{1 \leqq j<k \leqq n} x_{k} x_{j}\left\langle\chi_{\left[S_{k}, T_{k}\right]} \otimes f_{k}, \chi_{\left[S_{j}, T_{j}\right]} \otimes f_{j}\right\rangle\right) \\
= & \left\langle\Psi, W\left(x_{1} \chi_{\left[S_{1}, T_{1}\right]} \otimes f_{1}\right) \ldots W\left(x_{n} \chi_{\left[S_{n}, T_{n}\right]} \otimes f_{n}\right) \Psi\right\rangle .
\end{aligned}
$$

The uniformity of the convergence is proved as in Theorem (3.4) of [3].

\section{The Basic Estimates in the Fock Case}

As in the weak coupling limit, the gist of our analysis will be a detailed study of expressions of the form

$$
\begin{aligned}
& \int_{0}^{t / z^{2}} d t_{1} \int_{0}^{t_{1}} d t_{2} \ldots \int_{0}^{t_{n}-1} d t_{n} \\
& \quad \times\left\langle u \otimes W\left(z \int_{S / z^{2}}^{T / z^{2}} S_{u} f d u\right) \Phi,(-i)^{n} V\left(t_{1}\right) \ldots V\left(t_{n}\right) \cdot v \otimes W\left(z \int_{S^{\prime} / z^{2}}^{T^{\prime} / z^{2}} S_{u} f^{\prime} d u\right) \Phi\right\rangle
\end{aligned}
$$

with $n \in \mathbf{N}, S, T, S^{\prime}, T^{\prime} \in \mathbf{R}$ and $f, f^{\prime} \in K$, which arise from the expansion of $U_{t / z^{2}}$ using the iterated series. Using the explicit form of the interaction $V(t)$, i.e. (1.16), this expression can be written as

$$
\begin{aligned}
& \sum_{\varepsilon \in\{0,1\}^{n}} \int_{0}^{t / z^{2}} d t_{1} \int_{0}^{t_{1}} d t_{2} \ldots \int_{0}^{t_{n-1}} d t_{n} \\
& \times\left\langle W\left(z \int_{S / z^{2}}^{T / z^{2}} S_{u} f d u\right) \Phi, A^{+}\left(S_{t_{1}} g_{\varepsilon(1)}\right) A\left(S_{t_{1}} g_{1-\varepsilon(1)}\right) \ldots\right. \\
& \left.\quad \ldots A^{+}\left(S_{t_{n}} g_{\varepsilon(n)}\right) A\left(S_{t_{n}} g_{1-\varepsilon(n)}\right) \cdot W\left(z \int_{S^{\prime} / z^{2}}^{T^{\prime} / z^{2}} S_{u} f^{\prime} d u\right) \Phi\right\rangle \\
& \times\left\langle u, D_{\varepsilon(1)} \ldots D_{\varepsilon(n)} v\right\rangle .
\end{aligned}
$$

We introduce the notation $\Delta_{n}^{\varepsilon}(z, t)$ to denote the generic term in the sum (3.2). We shall need to identify the limit of the matrix elements $\Delta_{n}^{\varepsilon}(z, t)$ for any $\varepsilon \in\{0,1\}^{n}$, as $z \rightarrow 0$. Unfortunately, the only (essentially) way to achieve this explicit control is to bring the product of creators and annihilators in (3.2) into normally ordered form and then let the annihilators act on the coherent vector on the right and the creators on that on the left. The resulting expression is described in the following lemmata: 
Lemma (3.1). For each $n \in \mathbf{N}$ and $\varepsilon \in\{0,1\}^{n}$,

$$
\begin{aligned}
& \Delta_{n}^{\varepsilon}(z, t)=\sum_{d=0}^{n} \sum_{2 \leqq q_{1}<\ldots<q_{d} \leqq n} \sum_{\substack{1 \leqq p_{1}, \ldots, p_{d} \leqq n \\
\left|\left\{p_{h}\right\}_{h=1}^{d}\right|=d, p_{h}<q_{h}, h=1, \ldots, d}} z^{2 n-2 d} \\
& \times \int_{0}^{t / z^{2}} d t_{1} \int_{0}^{t_{1}} d t_{2} \ldots \int_{0}^{t_{n}-1} d t_{n} \\
& \times\left\langle W\left(z \int_{S / z^{2}}^{T / z^{2}} S_{u} f d u\right) \Phi, W\left(z \int_{S^{\prime} / z^{2}}^{T^{\prime} / z^{2}} S_{u} f^{\prime} d u\right) \Phi\right\rangle \\
& \times \prod_{h=1}^{d}\left\langle S_{t_{p_{h}}} g_{1-\varepsilon\left(p_{h}\right)}, S_{t_{q_{h}}} g_{\varepsilon\left(q_{h}\right)}\right\rangle \prod_{\alpha \in\{1, \ldots, n\} \backslash\left\{q_{h}\right\}_{h=1}^{d}} \int_{S / z^{2}}^{T / z^{2}}\left\langle S_{u} f, S_{t_{\alpha}} g_{\varepsilon(\alpha)}\right\rangle d u \\
& \times \prod_{\alpha \in\{1, \ldots, n\} \backslash\left\{p_{h}\right\}_{h=1}^{d}} \int_{S^{\prime} \mid z^{2}}^{T^{\prime} / z^{2}}\left\langle S_{t_{\alpha}} g_{1-\varepsilon(\alpha)}, S_{u} f^{\prime}\right\rangle d u
\end{aligned}
$$

with the convention that if $d=0$, then the sums over $q_{1}<\ldots<q_{d}$ and $p_{1}, \ldots, p_{d}$ and the product over $h$, in (3.3) are equal to 1.

Remark (3.2). In the following we shall frequently meet expressions with the same qualitative structure of the right-hand side of (3.3). In these expressions four types of scalar products appear:

(i) Scalar products arising from commutators of annihilators and creators. We call them $(g, g)$-terms and say that the corresponding operators have been used to produce scalar product.

(ii) Scalar products arising from the action of annihilators on coherent vectors: the $\left(g, f^{\prime}\right)$-terms.

(iii) Scalar products arising from the action of creators on coherent vectors: the $(f, g)$-terms.

(iv) The scalar product between the two initial coherent vectors, which remains after the action of the annihilators and creators.

It will be helpful for the reader to keep in mind a notation used coherently in this paper (and in fact throughout the whole series of papers [3-7, 21, 22]): the indices $q_{h}$ always label the creators and the $p_{h}$ the annihilators, which have been used to produce scalar products (by commutators).

To keep this in mind will help in giving an intuitive content to the long cues of summations which shall be found in the following.

Proof. For each $n \in \mathbf{N}, \varepsilon \in\{0,1\}^{n}$, we want to bring the product

$$
A^{+}\left(S_{t_{1}} g_{\varepsilon(1)}\right) A\left(S_{t_{1}} g_{1-\varepsilon(1)}\right) \ldots A^{+}\left(S_{t_{n}} g_{\varepsilon(n)}\right) A\left(S_{t_{n}} g_{1-\varepsilon(n)}\right)
$$

in Wick ordered form, i.e.

$$
\begin{gathered}
A^{+}\left(S_{t_{1}} g_{\varepsilon(1)}\right) A\left(S_{t_{1}} g_{1-\varepsilon(1)}\right) \ldots A^{+}\left(S_{t_{n}} g_{\varepsilon(n)}\right) A\left(S_{t_{n}} g_{1-\varepsilon(n)}\right) \\
\quad=\sum_{d=0}^{n} C(g, \varepsilon, d)\left(A^{+} \ldots\right)^{n-d} \cdot(A \ldots)^{n-d},
\end{gathered}
$$

where $C(g, \varepsilon, d)$ is a product of some scalar products of the $g_{j}$ and $d$ is the number of scalar products arising from the exchange of a creator with an annihilator at its left. Notice that, in the left-hand side of (3.4), the operator in the extreme right is an annihilator and the one on the extreme left a creator. Therefore, $n-d \geqq 1$, i.e. 
$d \leqq n-1$. For each $d$, we can choose $d$ creators among

$$
A^{+}\left(S_{t_{2}} g_{\varepsilon(2)}\right), \ldots, A^{+}\left(S_{t_{n}} g_{\varepsilon(n)}\right)
$$

which are used to produce scalar products with annihilators. Each choice determines a unique subset of $\{2, \ldots, n\}$, denoted $\left\{q_{1}, \ldots, q_{d}\right\}$. Clearly, we can suppose that

$$
q_{1}<\ldots<q_{d} .
$$

If the operators $A^{+}\left(S_{t_{\alpha}} g_{\varepsilon(\alpha)}\right)$ with $\alpha \in\{1, \ldots, n\} \backslash\left\{q_{h}\right\}_{h=1}^{d}$ have been moved to the left of the annihilation operators, it means that for each fixed $q_{h}$, there exists a $p_{h}<q_{h}$ such that the operator $A\left(S_{t_{p_{h}}} g_{1-\varepsilon\left(p_{h}\right)}\right)$ has been used to produce the scalar product $\left.\left\langle S_{t_{p_{h}}} g_{1-\varepsilon\left(p_{h}\right)}, S_{t_{q_{h}}} g_{\varepsilon\left(q_{h}\right)}\right)\right\rangle$. Therefore, the remaining set of annihilators is $\left\{A\left(S_{t_{\alpha}} g_{1-\varepsilon(\alpha)}\right) ; \alpha^{\prime} \in\{1, \ldots, n\} \backslash\left\{p_{h}\right\}_{h=1}^{d}\right\}$. Thus the right-hand side of (3.4) is equal to, with the same convention as in the statement of the lemma,

$$
\begin{gathered}
\sum_{d=0}^{n} \sum_{2 \leqq q_{1}<\ldots<q_{d} \leqq n} \sum_{\substack{1 \leqq p_{1}, \ldots, p_{d} \leqq n \\
\mid\left\{p_{h}\right\}_{h=1}^{d}=p_{h}=p_{h}<q_{h}, h=1, \ldots, d}} \prod_{h=1}^{d}\left\langle S_{t_{p}} g_{1-\varepsilon\left(p_{h}\right)}, S_{t_{q}} g_{\varepsilon\left(q_{h}\right)}\right\rangle \\
\quad \times \prod_{\alpha \in\{1, \ldots, n\} \backslash\left\{q_{h}\right\}_{h=1}^{d}} A^{+}\left(S_{t_{\alpha}} g_{\varepsilon(\alpha)}\right) \cdot \underbrace{}_{\alpha \in\{1, \ldots, n\} \backslash\left\{p_{h}\right\}_{h=1}^{d}} A\left(S_{t_{\alpha}} g_{1-\varepsilon(\alpha)}\right) .
\end{gathered}
$$

From (3.7), one deduces (3.3) by taking the matrix elements in the collective coherent vectors.

Notice that, because of the assumption (1.12), for each $h=1, \ldots, d$, if $1-\varepsilon\left(p_{h}\right)$ $\neq \varepsilon\left(q_{h}\right)$, then, $\left\langle S_{t_{p_{h}}} g_{1-\varepsilon\left(p_{h}\right)}, S_{t_{q_{h}}} g_{\varepsilon\left(q_{h}\right)}\right\rangle=0$. Therefore, many of the products in (3.3) are zero.

Now, proceeding as in [3], for each $n \in \mathbf{N}$ and $\varepsilon \in\{0,1\}^{n}$, in the quantity $\Delta_{n}^{\varepsilon}(z, t)$, we separate the terms which will be relevant in the limit $z \rightarrow 0$ (type I terms) from those which will vanish in the limit (type II terms). More precisely, in the summation (3.3) we call time-consecutive those terms for which $p_{h}=q_{h}-1$, i.e. those terms in which each scalar product arising from a commutator corresponds to a product of the form $A\left(S_{t_{j-1}} g_{\varepsilon}\right) \cdot A^{+}\left(S_{t_{j}} g_{\varepsilon}\right)$. With this notation we define

$$
\Delta_{n}^{\varepsilon}(z, t)=: I_{n}^{\varepsilon}(z, t)+I I_{n}^{\varepsilon}(z, t),
$$

where $I_{n}^{\varepsilon}(z, t)$ corresponds to the term in which the summation is for all timeconsecutive terms in (3.3), i.e.

$$
\begin{aligned}
I_{n}^{\varepsilon}(z, t):= & \sum_{m=0}^{k \wedge(n-k)} \sum_{2 \leqq q_{1}<\ldots<q_{m} \leqq n} z^{2 n-2 m} \int_{0}^{t / z^{2}} d t_{1} \int_{0}^{t_{1}} d t_{2} \ldots \int_{0}^{t_{n-1}} d t_{n} \\
& \times\left\langle W\left(z \int_{S / z^{2}}^{T / z^{2}} S_{u} f d u\right) \Phi, W\left(z \int_{S^{\prime} / z^{2}}^{T T^{\prime} / z^{2}} S_{u} f^{\prime} d u\right) \Phi\right\rangle \\
& \times \prod_{h=1}^{m}\left\langle g_{\varepsilon\left(q_{h}-1\right)}, S_{\left(t_{q_{h}}-t_{q_{h}}-1\right)} g_{\varepsilon\left(q_{h}\right)}\right\rangle \\
& \times \underset{\alpha \in\{1, \ldots, n\} \backslash\left\{q_{h}\right\}_{h=1}^{m}}{ } \int_{S / z^{2}}^{T / z^{2}}\left\langle S_{u} f, S_{t_{\alpha}} g_{\varepsilon(\alpha)}\right\rangle d u \\
& \times \prod_{\alpha \in\{1, \ldots, n\} \backslash\left\{q_{h}-1\right\}_{h=1}^{m}} \int_{S^{\prime} / z^{2}}^{T^{\prime} / z^{2}}\left\langle S_{t_{\alpha}} g_{1-\varepsilon(\alpha)}, S_{u} f^{\prime}\right\rangle d u
\end{aligned}
$$

and $I I_{n}^{\varepsilon}(z, t)$ corresponds to the term in which the summation is for all non-time consecutive terms in (3.3), i.e. there exists some $h=1, \ldots, m$, such that $p_{h}<q_{h}-1$.

Our first result is that the type $I I$ terms tend to zero as $z$ tends to zero. 
Lemma (3.3). For each $n \in \mathbf{N}, \varepsilon \in\{0,1\}^{n}$,

$$
\lim _{z \rightarrow 0} I I_{n}^{\varepsilon}(z, t)=0 \text {. }
$$

Proof. First of all, for $n \in \mathbf{N}, \varepsilon \in\{0,1\}^{n}$, we majorize with constants all the scalar products in $I I_{n}^{\varepsilon}(z, t)$ which do not come from commutators. Therefore, the modulus of $I I_{n}^{\varepsilon}(z, t)$ is majorized by

$$
\begin{aligned}
& \sum_{m=0}^{n-1} \sum_{2 \leqq q_{1}<\ldots<q_{m} \leqq n} \sum_{\left(q_{1}, p_{1}, \ldots, q_{m}, p_{m}\right)}^{\prime} C_{1}^{n} \cdot z^{2 n-2 m} \\
& \times \int_{0}^{t / z^{2}} d t_{1} \int_{0}^{t_{1}} d t_{2} \ldots \int_{0}^{t_{n}-1} d t_{n} \prod_{h=1}^{m}\left|\left\langle S_{t_{p_{h}}} g, S_{t_{q_{h}}} g\right\rangle\right|,
\end{aligned}
$$

where $\sum_{\left(q_{1}, p_{1}, \ldots, q_{m}, p_{m}\right)}^{\prime}$ means the sum for all $1 \leqq p_{1}, \ldots, p_{m} \leqq n$ with $\left|\left\{p_{h}\right\}_{h=1}^{m}\right|=m$, $p_{h}<q_{h}, h=1, \ldots, m$ and $p_{h}<q_{h}-1$, for some $h=1, \ldots, m ; g=g_{0}$ or $g_{1}$ and

$$
C_{1}:=\max _{F=f, f^{\prime}, G=g_{0}, g_{1}} \int_{-\infty}^{\infty}\left|\left\langle G, S_{t} F\right\rangle\right| d t .
$$

The idea of the proof is to exploit the type- $I I$ hypothesis, i.e. the existence of some pair $\left(p_{h}, q_{h}\right)$ with $q_{h}-p_{h} \geqq 2$, to majorize each term of the sum (3.10) with an expression of the form

$$
\begin{aligned}
& C_{2}^{n} \cdot \int_{0}^{t} d t_{1} \int_{0}^{t_{1}} d t_{2} \ldots \int_{0}^{t_{q_{1}}-2} d t_{q_{1}-1} \int_{0}^{t_{q_{1}}-1} d t_{q_{1}+1} \cdots
\end{aligned}
$$

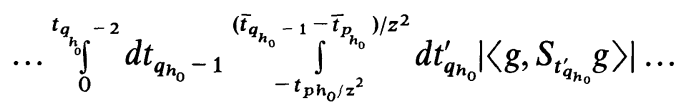

$$
\begin{aligned}
& \cdots \int_{0}^{t_{q_{m}-2}} d t_{q_{m}-1} \int_{0}^{t_{q_{m}-1}} d t_{q_{m}+1} \cdots \int_{0}^{t_{n-1}} d t_{n},
\end{aligned}
$$

where $h_{0}, \bar{t}_{q_{h_{0}}-1}, \bar{t}_{p_{h_{0}}}$ will be defined below, $C_{2}$ is a constant and $\bar{t}_{q_{h_{0}}-1}-\bar{t}_{p_{h_{0}}}<0$ for almost all $t_{q_{h_{0}}-1}, t_{p_{h_{0}}}$. The integrability of $t \rightarrow\left|\left\langle g, S_{t} g\right\rangle\right|$ will then imply that (3.12) tends to zero as $z \rightarrow 0$.

This majorization is achieved through a sequence of changes of variables and of majorizations, according to the scheme described below, which heavily uses the specific form of the interaction (and, of course, the type $I I$ assumption). Let

$$
h_{0}:=\min \left\{h: q_{h}-p_{h} \geqq 2, h=1, \ldots, m\right\}
$$

and let $\Delta_{n}^{\varepsilon}\left(z, t,\left\{q_{h}, p_{h}\right\}_{h=1}^{m}\right)$ denote the generic term of the sum (3.10). Then,

$$
\begin{aligned}
& \left|\Delta_{n}^{\varepsilon}\left(z, t,\left\{q_{h}, p_{h}\right\}_{h=1}^{m}\right)\right| \\
& \leqq C_{1}^{n} \cdot z^{-2 m} \int_{0}^{t} d t_{1} \int_{0}^{t_{1}} d t_{2} \ldots \int_{0}^{t_{n-1}} d t_{n} \prod_{h=1}^{m}\left|\left\langle g, S_{\left(t_{q_{h}}-t_{p_{h}}\right) / z^{2}} g\right\rangle\right| \\
& =C_{1}^{n} \cdot z^{-2(m-1)} \int_{0}^{t} d t_{1} \int_{0}^{t_{1}} d t_{2} \cdots \int_{0}^{t_{q_{1}}-2} d t_{q_{1}-1} \int_{-t_{p_{1} / z^{2}}}^{\left(t_{q_{1}}-1-t_{p_{1}}\right) / z^{2}} d t_{q_{1}}^{\prime}\left|\left\langle g, S_{t_{q_{1}}} g\right\rangle\right| \\
& \quad \times \int_{0}^{t_{p_{1}}+z^{2} t_{q_{1}}^{\prime}} d t_{q_{1}+1} \cdots \int_{0}^{t_{n}-1} d t_{n} \prod_{h=2}^{m}\left|\left\langle g, S_{\left(t_{q_{h}}-t_{p_{h}}\right) / z^{2}} g\right\rangle\right|
\end{aligned}
$$

where, if for some $h=2, \ldots, m, p_{h}=q_{1}$, then $t_{p_{h}}$ means $t_{p_{1}}+z^{2} t_{q_{1}}^{\prime}$. Since $\left.t_{q_{1}}^{\prime} \in\left[-t_{p_{1}} / z^{2},\left(t_{q_{1}-1}-t_{p_{1}}\right) / z^{2}\right)\right)$, one has $t_{p_{1}}+z^{2} t_{q_{1}}^{\prime} \in\left[0, t_{q_{1}-1}\right)$, thus, replacing 
20

L. Accardi and Y. G. Lu

$t_{p_{1}}+z^{2} t_{q_{1}}^{\prime}$ by $t_{q_{1}-1}$, we majorize the integral, ie.:

$$
\begin{aligned}
& \left|\Delta_{n}^{\varepsilon}\left(z, t,\left\{q_{h}, p_{h}\right\}_{h=1}^{m}\right)\right| \\
& \leqq C_{1}^{n} \cdot z^{-2(m-1)} \int_{0}^{t} d t_{1} \int_{0}^{t_{1}} d t_{2} \ldots \int_{0}^{t_{q_{1}}-2} d t_{q_{1}-1} \int_{-t_{p_{1} / z^{2}}}^{\left(t_{q_{1}-1}-t_{p_{1}}\right) / z^{2}} d t_{q_{1}}^{\prime}\left|\left\langle g, S_{t_{q_{1}}} g\right\rangle\right| \\
& \quad \times \int_{0}^{t_{q_{1}-1}} d t_{q_{1}+1} \ldots \int_{0}^{t_{n-1}} d t_{n} \prod_{h=2}^{m}\left\langle g, S_{\left(t_{q_{h}}-t_{p_{h}}\right) / z^{2}} g\right\rangle \mid .
\end{aligned}
$$

Put $t_{q_{2}}^{\prime}=\left(t_{q_{2}}-t_{p_{2}}\right) / z^{2}$, then,

$$
\begin{aligned}
& \left|\Delta_{n}^{\varepsilon}\left(z, t,\left\{q_{h}, p_{h}\right\}_{h=1}^{m}\right)\right| \\
& \leqq C_{1}^{n} \cdot z^{-2(m-2)} \int_{0}^{t} d t_{1} \int_{0}^{t_{1}} d t_{2} \ldots \int_{0}^{t_{q_{1}}-2} d t_{q_{1}-1} \int_{-t_{p_{1} / z^{2}}}^{\left(t_{q_{1}}-1-t_{p_{1}}\right) / z^{2}} d t_{q_{1}}^{\prime}\left|\left\langle g, S_{t_{q_{1}}^{\prime}} g\right\rangle\right| \\
& \times \int_{0}^{t_{q_{1}}-1} d t_{q_{1}+1} \ldots \int_{-t_{p / z}}^{\left(\bar{t}_{q_{2}-1}-t_{p_{2}}\right) / z^{2}} d t_{q_{2}}^{\prime}\left|\left\langle g, S_{t_{q_{2}}^{\prime}} g\right\rangle\right| \\
& \times \int_{0}^{t_{p_{2}}+z^{2} t_{q_{2}}^{\prime}} d t_{q_{2}+1} \cdots \int_{0}^{t_{n-1}} d t_{n} \prod_{h=3}^{m}\left|\left\langle g, S_{\left(t_{q_{h}}-t_{p_{h}}\right) / z^{2}} g\right\rangle\right|,
\end{aligned}
$$

where

$$
\bar{t}_{q_{2}-1}=\left\{\begin{array}{lll}
t_{q_{2}-1}, & \text { if } & q_{2}>q_{1}+1 \\
t_{q_{2}-2}, & \text { if } & q_{2}=q_{1}+1
\end{array}\right.
$$

Since $\left.t_{q_{2}}^{\prime} \in\left[-t_{p_{2} / z^{2}},\left(\bar{t}_{q_{2}-1}-t_{p_{2}}\right) / z^{2}\right)\right)$, one has $t_{p_{2}}+z^{2} t_{q_{2}}^{\prime} \in\left[0, \bar{t}_{q_{2}-1}\right)$. So by the same argument as after (3.13), it can be obtained that

$$
\begin{aligned}
& \left|\Delta_{n}^{\varepsilon}\left(z, t,\left\{q_{h}, p_{h}\right\}_{h=1}^{m}\right)\right| \\
& \leqq \\
& \quad C_{1}^{n} \cdot z^{-2(m-2)} \int_{0}^{t} d t_{1} \int_{0}^{t_{1}} d t_{2} \ldots \int_{0}^{t_{q_{1}}-2} d t_{q_{1}-1} \int_{-t_{p_{1} / z^{2}}}^{\left(t_{q_{1}}-1-t_{p_{1}}\right) / z^{2}} d t_{q_{1}}^{\prime}\left|\left\langle g, S_{t_{q_{1}}} g\right\rangle\right| \\
& \quad \times \int_{0}^{t_{q_{1}}-1} d t_{q_{1}+1} \ldots \int_{\left(t_{q_{2}-1}-t_{p_{2}}\right) / z^{2}} \int_{-t_{p_{1}} / z^{2}} d t_{q_{2}}^{\prime}\left|\left\langle g, S_{t_{q_{2}}} g\right\rangle\right| \\
& \quad \times \int_{0}^{\bar{t}_{q_{2}}-1} d t_{q_{2}+1} \ldots \int_{0}^{t_{n-1}} d t_{n} \prod_{h=3}^{m}\left|\left\langle g, S_{\left(t_{q_{h}}-t_{p_{h}}\right) / z^{2}} g\right\rangle\right| .
\end{aligned}
$$

Iterating the procedure, we obtain

$$
\begin{aligned}
& \left|\Delta_{n}^{\varepsilon}\left(z, t,\left\{q_{h}, p_{h}\right\}_{h=1}^{m}\right)\right| \\
& \leqq C_{1}^{n} \cdot C_{1}^{\prime} \cdot \int_{0}^{t} d t_{1} \int_{0}^{t_{1}} d t_{2} \ldots \int_{0}^{t_{q_{1}}-2} d t_{q_{1}-1} \int_{-t_{p_{1}} / z^{2}}^{\left(t_{q_{1}}-1-t_{p_{1}}\right) / z^{2}} d t_{q_{1}}^{\prime}\left|\left\langle g, S_{t_{q_{1}}} g\right\rangle\right| \\
& \times \int_{0}^{t_{q_{1}}-1} d t_{q_{1}+1} \ldots \int_{0}^{t_{q_{2}}-2} d t_{q_{2}-1} \int_{-\bar{t}_{p_{2} / z^{2}}}^{\left(\bar{t}_{q_{2}-1}-\bar{t}_{p_{2}}\right) / z^{2}} d t_{q_{2}}^{\prime}\left|\left\langle g, S_{t_{q_{2}}} g\right\rangle\right| \\
& \times \int_{0}^{\bar{t}_{q_{2}}-1} d t_{q_{2}+1} \cdots \int_{0}^{t_{q_{m}-2}} d t_{q_{m}-1} \int_{-\bar{t}_{p_{m} / z^{2}}}^{\left(\bar{t}_{q_{m_{m}}-1}-\bar{t}_{p_{m}}\right) / z^{2}} d t_{q_{m}}^{\prime}\left|\left\langle g, S_{t_{q_{m}}^{\prime}} g\right\rangle\right| \\
& \times \int_{0}^{\bar{t}_{q_{m}-1}} d t_{q_{m}+1} \cdots \int_{0}^{t_{n-1}} d t_{n}
\end{aligned}
$$


with

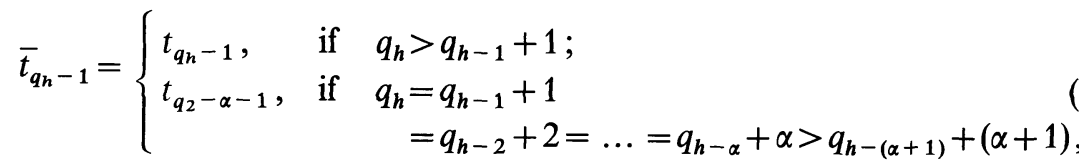

$$
\begin{aligned}
& \bar{t}_{p_{h}}=\left\{\begin{array}{lll}
t_{q_{\alpha}}^{\prime}, & \text { if } & p_{h}=q_{\alpha} \\
t_{p_{h}}, & \text { if } & p_{h} \notin\left\{q_{h}\right\}_{h=1}^{m} .
\end{array}\right.
\end{aligned}
$$

Notice that there exists always an $\alpha \in \mathbf{N}$, such that $\bar{t}_{q_{h_{0}}-1}=t_{q_{h_{0}}-\alpha-1}$. Moreover, by the definition of $h_{0}$, it follows that $p_{h_{0}}<q_{h_{0}}-\alpha-1$. In fact, if $\alpha=0$, this follows from the definition of $h_{0}$; if $\alpha>0$, then (3.19) and the definition of $h_{0}$ imply that, for any $0 \leqq \beta \leqq \alpha, q_{h_{0}}-\beta-1=q_{h_{0}-\beta}-1=p_{h_{0}-\beta}$. Therefore, it must be that $p_{h_{0}}<p_{h_{0}-\beta}$ $=q_{h_{0}-\beta}-1$, since at each time there is only one annihilator. Summing up: we have proved that $\bar{t}_{q_{h 0}-1}-\bar{t}_{p_{h 0}}<0$ almost everywhere. Now we majorize all the $d t_{q_{h}}^{\prime}$-integrals in (3.18), with the exception of the $d t_{q_{h_{0}}}^{\prime}$-one, by integrating over the whole line. This leads to an expression of the form (3.12) and therefore, the proof is concluded.

Having proved that the type $I I$ terms tend to zero, we shall now compute the limit of the type $I$ terms. The resulting lengthy expression does not give, by itself, particular insight. However, it will play a crucial role in the deduction of the equation satisfied by the limit. After the statement of Lemma (3.4) we shall introduce some comments in order to clarify the meaning and the origin of the various pieces.

Lemma (3.4). For each $n \in \mathbf{N}, \varepsilon \in\{0,1\}^{n}$,

$$
\begin{aligned}
& I_{n}^{\varepsilon}(t):=\lim _{z \rightarrow 0} I_{n}^{\varepsilon}(z, t)=\sum_{m=0}^{k \wedge(n-k)} \sum_{2 \leqq q_{1}<\ldots<q_{m} \leqq n} \prod_{h=1}^{m}\left(g_{\varepsilon\left(q_{h}-1\right)} \mid g_{\varepsilon\left(q_{h}\right)}\right)- \\
& \times \underset{0 \leqq t_{n} \leqq \ldots \leqq \widehat{t_{q_{m}}} \leqq \ldots \leqq \widehat{t_{q_{1}}} \leqq \ldots \leqq t_{1} \leqq t}{ } d t_{1} \ldots \widehat{d t_{q_{1}}} \ldots \widehat{d t_{q_{m}}} \ldots d t_{n} \\
& \times \prod_{\alpha \in\{1, \ldots, n\} \backslash\left\{q_{h}\right\}_{h=1}^{m}}\left(f \mid g_{\varepsilon(\alpha)}\right) \cdot \chi_{[S, T]}\left(t_{\alpha}\right) \\
& \times\left(g_{1-\varepsilon\left(q_{r_{1}}\right)} \mid f^{\prime}\right) \cdot \chi_{\left[S^{\prime}, T^{\prime}\right]}\left(t_{q_{1}-1}\right) \cdot \prod_{\alpha \in\left\{1, \ldots, q_{1}-2\right\}}\left(g_{1-\varepsilon(\alpha)} \mid f^{\prime}\right) \cdot \chi_{\left[S^{\prime}, T^{\prime}\right]}\left(t_{\alpha}\right) \\
& \times\left(g_{1-\varepsilon\left(q_{r_{2}}\right)} \mid f^{\prime}\right) \cdot \chi_{\left[S^{\prime}, T^{\prime}\right]}\left(t_{q_{1}+1-1}\right) \cdot \prod_{\alpha \in\left\{q_{r_{1}}+1, \ldots, q_{r_{1}+1}-2\right\}}\left(g_{1-\varepsilon(\alpha)} \mid f^{\prime}\right) \cdot \chi_{\left[S^{\prime}, T^{\prime}\right]}\left(t_{\alpha}\right) \\
& \times\left(g_{1-\varepsilon\left(q_{r_{x}}\right)}|f|^{\prime}\right) \cdot \chi_{\left[S^{\prime}, T^{\prime}\right]}\left(t_{q_{x-1}-1}\right) \cdot \prod_{\alpha \in\left\{q_{r_{x-1}}+1, \ldots, q_{r_{x-1}+1}-2\right\}}\left(g_{1-\varepsilon(\alpha)} \mid f^{\prime}\right) \cdot \chi_{\left[S^{\prime}, T^{\prime}\right]}\left(t_{\alpha}\right) \\
& \times \prod_{\alpha \in\left\{q_{r x}+1, \ldots, n\right\}}\left(g_{1-\varepsilon(\alpha)} \mid f^{\prime}\right) \cdot \chi_{\left[S^{\prime}, T^{\prime}\right]}\left(t_{\alpha}\right) \cdot\left\langle W\left(\chi_{[S, T]} \otimes f\right) \Psi, W\left(\chi_{\left[S,, T^{\prime}\right]} \otimes f^{\prime}\right) \Psi\right\rangle,
\end{aligned}
$$

where $\hat{t}$ means that the $t$ is absent, $x$ (i.e. the total number of chains), and the numbers $\left\{r_{h}\right\}_{h=1}^{x}$ (i.e. the lengths of the single chain) are uniquely determined by the prescription

$$
\left\{q_{h}\right\}_{h=1}^{m+m^{\prime}}=\left\{q_{h}\right\}_{h=1}^{r_{1}} \cup\left\{q_{h}\right\}_{h=r_{1}+1}^{r_{2}} \cup \ldots \cup\left\{q_{h}\right\}_{h=r_{x-1}+1}^{r_{x}}
$$

and

$$
q_{r_{y}+1}=q_{r_{y}+2}-1=q_{r_{y}+3}-2=\ldots=q_{r_{y+1}}-\left(r_{y+1}-r_{y}-1\right)<q_{r_{y+1}+1}-\left(r_{y+1}-r_{y}\right),
$$


with

$$
y=0,1, \ldots, x-1, \quad r_{0}=0 .
$$

Remark. The conditions (3.22), (3.23a), (3.23b) mean that we split the ordered sequence $\left\{q_{h}\right\}_{h=1}^{m}$ into ordered subsequences which are maximal with respect to the property that $q_{h+1}=q_{h}+1$ for all elements in the subsequence (a subsequence may contain a single element). The role of these ordered subsequences comes from the change of variables (3.25) below and will be explained during the proof.

Proof. With the above notations the term $I_{n}^{\varepsilon}(z, t)$, defined by (3.8), can be written as a sum whose indices are the same as in the right-hand side of (3.21) and whose generic term is the product of

$$
\left\langle W\left(z \int_{S / z^{2}}^{T / z^{2}} S_{u} f d u\right) \Phi, W\left(z \int_{S^{\prime} / z^{2}}^{T^{\prime} / z^{2}} S_{u} f^{\prime} d u\right) \Phi\right\rangle
$$

times

$$
\begin{aligned}
& \left.z^{2 n-2 m} \int_{0}^{t / z^{2}} d t_{1} \int_{0}^{t_{1}} d t_{2} \ldots \int_{0}^{t_{n}-1} d t_{n} \prod_{h=1}^{m}\left\langle g_{\varepsilon\left(q_{h}-1\right)}, S_{\left(t_{q_{h}}-t_{q_{h}}-1\right)} g_{\varepsilon\left(q_{h}\right)}\right)\right\rangle \\
& \quad \times \prod_{\alpha \in\{1, \ldots, n\} \backslash\left\{q_{h}\right\}_{h=1}^{m}} \int_{S / z^{2}}^{T / z^{2}}\left\langle S_{u} f, S_{t_{\alpha}} g_{\varepsilon(\alpha)}\right\rangle d u . \prod_{\alpha \in\{1, \ldots, n\}\left\{\left\{q_{h}-1\right\}_{h=1}^{m}\right.} \int_{S^{\prime} / z^{2}}^{T^{\prime} / z^{2}}\left\langle S_{t_{\alpha}} g_{1-\varepsilon(\alpha)}, S_{u} f^{\prime}\right\rangle
\end{aligned}
$$

With the change of variable $z^{2} t_{j} \hookrightarrow t_{j}$ for each $j=1, \ldots, n$, the expression (3.24) becomes

$$
\begin{aligned}
& z^{-2 m} \int_{0}^{t} d t_{1} \int_{0}^{t_{1}} d t_{2} \ldots \int_{0}^{t_{n}-1} d t_{n} \cdot \prod_{h=1}^{m}\left\langle g_{\varepsilon\left(q_{h}-1\right)}, S_{\left(t_{q_{h}}-t_{q_{h}}-1\right) / z^{2}} g_{\varepsilon\left(q_{h}\right)}\right\rangle \\
& \times \prod_{\alpha \in\{1, \ldots, n\} \backslash\left\{q_{h}\right\}_{h=1}^{m} \cdot \int_{S / z^{2}}^{T / z^{2}}\left\langle S_{u} f, S_{t_{\alpha} / z^{2}} g_{\varepsilon(\alpha)}\right\rangle d u} \underbrace{}_{\alpha \in\{1, \ldots, n\} \backslash\left\{q_{h}-1\right\}_{h=1}^{m}} \int_{S^{\prime} / z^{2}}^{T^{\prime} / z^{2}}\left\langle S_{t_{\alpha} / z^{2}} g_{1-\varepsilon(\alpha)}, S_{u} f^{\prime}\right\rangle d u .
\end{aligned}
$$

In order to compute (3.24a), it is natural to make the change of variables:

$$
t_{\alpha}^{\prime}=\left\{\begin{array}{lll}
t_{\alpha}, & \text { if } & \alpha \in\{1, \ldots, n\} \backslash\left\{q_{h}\right\}_{h=1}^{m+m^{\prime}} \\
\left(t_{q_{h}}-t_{q_{h}-1}\right) / z^{2}, & \text { if } & \alpha \in\left\{q_{h}\right\}_{h=1}^{m+m^{\prime}}
\end{array}\right.
$$

which will affect only those times $t_{j}$ corresponding to the creation operators which have produced a scalar product by commutation with an annihilator consecutively later.

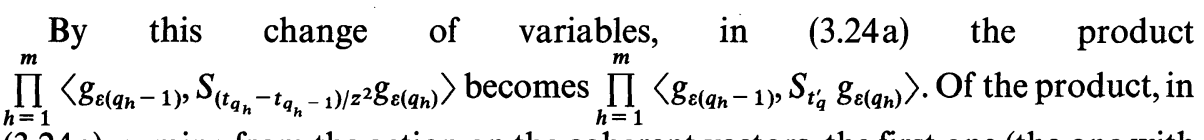
(3.24a), coming from the action on the coherent vectors, the first one (the one with the $f$-test function) does not change. Moreover, a simple computation (put $v:=u-t_{\alpha} / z^{2}$ ) shows that this term tends to

$$
\prod_{\alpha \in\{1, \ldots, n\} \backslash\left\{q_{h}\right\}_{h=1}^{m}}\left(f \mid g_{\varepsilon(\alpha)}\right) \cdot \chi_{[S, T]}\left(t_{\alpha}\right)
$$

which is the $(f \mid g)$-type product in (3.21). 
The basic idea to explain the remaining products in (3.21) is that one should know how the change of variables (3.25) influences the integrals corresponding to the intervals $\left[S^{\prime} / z^{2}, T^{\prime} / z^{2}\right]$. To clarify the situation we begin to consider the first chain, i.e.

$$
q_{1}<q_{1}+1=q_{2}<q_{2}+1=q_{3}<\ldots<q_{r_{1}}-1=q_{r_{1}-1}<q_{r_{1}}<q_{r_{1}+1}-1
$$

(notice the last strict inequality!). The integrals corresponding to this chain are:

$$
\begin{aligned}
z^{2 r_{1}} & \int_{0}^{t} d t_{1} \ldots \int_{0}^{t_{q_{1}}-1} d t_{q_{1}} \int_{0}^{t_{q_{1}}} d t_{q_{2}} \int_{0}^{t_{q_{2}}} d t_{q_{3}} \ldots \int_{0}^{t_{q_{r_{1}-1}}} d t_{q_{r_{1}}} \int_{0}^{t_{r_{1}}} d t_{q_{r_{1}}+1} \ldots \\
& \ldots \prod_{h=1}^{r_{1}}\left\langle g_{\varepsilon\left(q_{h}-1\right)}, S_{\left(t_{q_{h}}-t_{q_{h}}-1\right)} g_{\varepsilon\left(q_{n}\right)}\right\rangle \\
& \times \prod_{h=1}^{q_{1}-2} \int_{S^{\prime} / z^{2}}^{T^{\prime} / z^{2}}\left\langle S_{t_{h}} g_{1-\varepsilon(h)}, S_{u} f^{\prime}\right\rangle d u \\
& \times \int_{S^{\prime} / z^{2}}^{T^{\prime} / z^{2}}\left\langle S_{t_{q_{r_{1}}} / z^{2}} g_{1-\varepsilon\left(q_{r_{1}}\right)}, S_{u} f^{\prime}\right\rangle d u .
\end{aligned}
$$

The change of variables (3.25) brings the variable $t_{q_{1}} / z^{2}$ into

$$
t_{q_{1}-1}^{\prime} / z^{2}+t_{q_{1}}^{\prime}+\ldots+t_{q_{1}-1}^{\prime} \text {. }
$$

By the change of variable $v=u-\left(t_{q_{1}-1}^{\prime} / z^{2}+t_{q_{1}}^{\prime}+\ldots+t_{q_{r_{1}}-1}^{\prime}\right)$, the last integral in (3.26) becomes

$$
\left.\begin{array}{l}
\left(T^{\prime}-t_{q_{1}-1}^{\prime}\right) / z^{2}-\left(t_{q_{1}}^{\prime}+\ldots+t_{q_{1}}^{\prime}-1\right) \\
\left(S^{\prime}-t_{q_{1}-1}^{\prime}\right) / z^{2}-\left(t_{q_{1}}^{\prime}+\ldots+t_{q_{1}}^{\prime}-1\right)
\end{array} g_{1-\varepsilon\left(q_{r_{1}}\right)}, S_{v} f^{\prime}\right\rangle d v
$$

which, as $z \rightarrow 0$, converges to

$$
\left(g_{1-\varepsilon\left(q_{r_{1}}\right)} \mid f^{\prime}\right) \cdot \chi_{\left[S^{\prime}, T^{\prime}\right]}\left(t_{q_{1}-1}^{\prime}\right) .
$$

On the other hand, in the last second integral in (3.21), one has $h \leqq q_{1}-2$. Therefore, the change of variables (3.25) does not affect these variables $t_{h}$. It is to see, by the change of variable $v=u-t_{h}^{\prime} / z^{2}$, that for each $h=1, \ldots, q_{1}-2$ the last second integral tends, as $z \rightarrow 0$, to

$$
\left(g_{1-\varepsilon(h)} \mid f^{\prime}\right) \cdot \chi_{\left[S^{\prime}, T^{\prime}\right]}\left(t_{h}^{\prime}\right) \text {. }
$$

Similar arguments apply to all the other chains $q_{r_{y}+1}, \ldots, q_{r_{y+1}}$ $(y=0,1, \ldots, x-1)$, and this justifies all the remaining products in (3.21).

It remains to justify the $\prod_{h=1}^{m}\left(g_{1-\varepsilon\left(q_{h}-1\right)} \mid g_{\varepsilon\left(q_{h}\right)}\right)$ factor in (3.21) and the (subsequent) omission of the $t_{q_{h}}$-variables $(h=1, \ldots, m)$ from the integration in the same formula. That is, we have to prove that for each $y=0,1, \ldots, x-1$,

$$
\begin{gathered}
z^{-2\left(r_{y+1}-r_{y}\right)} \ldots \int_{0}^{t q_{r_{y}}-2} d t_{q_{r_{y}}-1} \int_{0}^{t_{q_{r_{y}}-1}} d t_{q_{r y}} \ldots \int_{0}^{t_{q_{r y+1}}} d t_{q_{r_{y+1}}+1} \ldots \\
\ldots \prod_{h=r_{y}}^{r_{y+1}}\left\langle g_{1-\varepsilon\left(q_{h}-1\right)}, S_{\left(t_{q_{h}}-t_{q_{h}}-1\right) / z^{2}} g_{\varepsilon\left(q_{h}\right)}\right\rangle
\end{gathered}
$$

converges, as $z \rightarrow 0$, to

$$
\ldots \int_{0}^{t_{q_{r_{y}}-2}} d t_{q_{r_{y}}-1} \int_{0}^{t_{q_{r_{y}}-1}} d t_{q_{r_{y+1}}+1} \ldots \prod_{h=r_{v}}^{r_{y+1}}\left(g_{1-\varepsilon\left(q_{h}-1\right)} \mid g_{\varepsilon\left(q_{h}\right)}\right)_{-} .
$$


In fact, the change of variables (3.25) implies that $t_{q_{h}}=z^{2} t_{q_{h}}^{\prime}+t_{q_{h}-1}^{\prime}, h=1, \ldots, m$, so that the expression (3.28) becomes

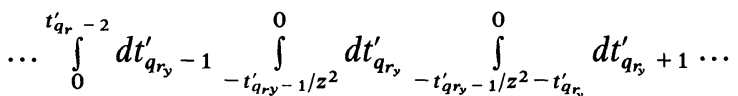

$$
\begin{aligned}
& \cdots t_{-q_{r_{y}}-1 / z^{2}-t_{q_{y}}^{\prime}-\ldots-t_{q_{y+1}}^{\prime}}^{0} d t_{q_{r_{y}+1}}^{\prime} \ldots \int_{0}^{t_{q_{r_{y}}-1}^{\prime}+z^{2}\left(t_{q_{r_{y}}}^{\prime}+\ldots+t_{q_{r_{y+1}}}\right)} d t_{q_{r_{y+1}}}^{\prime} \int_{0} \ldots \\
& \ldots \prod_{h=r_{y}}^{r_{y+1}}\left\langle g_{1-\varepsilon\left(q_{h}-1\right)}, S_{t_{q_{h}}^{\prime}} g_{\varepsilon\left(q_{h}\right)}\right\rangle \text {. }
\end{aligned}
$$

Notice that the $z^{2}$-dependent terms in (3.29) are of two types:

$$
\int_{0}^{t_{\alpha}^{\prime}+z^{2}(\ldots)}, \text { or } \int_{-t_{\alpha}^{\prime} / z^{2}-(\ldots)}^{0}
$$

In the limit $\underset{z \rightarrow 0}{z}$ the integrals of the form $\int_{0}^{t_{\alpha}^{\prime}+z^{2}(\ldots)}$ converge to $\int_{0}^{t_{\alpha}^{\prime}}$ and the integrals of the form $\int_{-t_{\alpha}^{\prime} / z^{2}-(\ldots)}^{0}$ give rise to the factor $\left(g \mid g^{\prime}\right)_{-}$(where $g, g^{\prime}$ denote either $g_{0}$ or $g_{1}$ ), and the corresponding integration variable disappears. The limiting is justified by dominated convergence and the following, easily verified, fact: for each $f_{1}, f_{2} \in L^{1}(\mathbf{R}), t>0$ and $T \in \mathbf{R} \cup\{+\infty\}$,

$$
\int_{-t / z}^{T}\left|f_{1}\left(s_{1}\right)\right| d s_{1} \int_{-t / z-s_{1}}^{T}\left|f_{2}\left(s_{2}\right)\right| d s_{2} \rightarrow \int_{-\infty}^{T}\left|f_{1}\left(s_{1}\right)\right| d s_{1} \int_{-\infty}^{T}\left|f_{2}\left(s_{2}\right)\right| d s_{2} .
$$

\section{The Uniform Estimate}

In Sect. 3, for each $n \in \mathbf{N}$, we have computed the limit, as $z \rightarrow 0$, of the $n^{\text {th }}$ term of the iterated series. In this section, we shall prove some uniform estimates on these terms, which will allow to take the limit of the series term by term. Due to the particular form of the interaction (1.9), the bounds will be strongly dependent on the test functions $g_{0}, g_{1}$ [cf. (4.4) below], contrary to what happens in [3].

As in the weak coupling limit case, the uniform estimate is based on the following Pulé type inequality which has been proved in [21, 22]:

Theorem (4.1). Let $f: \mathbf{R} \rightarrow \mathbf{R}_{+}$be a positive integrable symmetric function, for each $n \in \mathbf{N}, 1 \leqq m \leqq n-1,2 \leqq q_{1}<\ldots<q_{m} \leqq n, 1 \leqq p_{1}<\ldots<p_{m} \leqq n-1, p_{h}<q_{h}, h=1, \ldots, m$, one has

$\lambda^{-2 m} \int_{0}^{t} d t_{1} \ldots \int_{0}^{t_{n}-1} d t_{n} \sum_{\sigma \in s_{m}} \prod_{h=1}^{m} f\left(\frac{t_{p_{Q}(h)}-t_{q_{h}}}{\lambda^{2}}\right) \leqq \frac{t^{n-m}}{(n-\bar{m}) !(\bar{m}-m) !}\left[\int_{-\infty}^{0} f(t) d t\right]^{m}$,

where $\bar{m}:=\left|\left\{p_{h}, q_{h}\right\}_{h=1}^{m}\right|, \mathscr{S}_{m}$ denote the group of permutations on $m$ elements and

$$
\mathscr{S}_{m}^{0}:=\left\{\sigma \in \mathscr{S}_{m}: p_{\sigma(h)}<q_{h} ; h=1, \ldots, m\right\} .
$$

In the following, we shall use the notation

$$
\left\|g_{\varepsilon}\right\|_{-}^{2}:=\int_{-\infty}^{0}\left|\left\langle g_{\varepsilon}, S_{u} g_{\varepsilon}\right\rangle\right| d u, \quad \varepsilon=0,1
$$


Theorem (4.2) (The Uniform Estimate). For each $n \in \mathbf{N}$, the matrix element (3.1), corresponding to the $n^{\text {th }}$ term of the iterated series is majorized, in modulus, by

$$
16^{n} \cdot \max _{0 \leqq m \leqq n-1} \frac{t^{n-m}}{(n-m) !} \cdot\left(\left\|g_{0}\right\|_{-} \vee\left\|g_{1}\right\|_{-}\right)^{2 m}\left[\max _{F=f, f^{\prime} ; G=g_{0}, g_{1}} \int_{-\infty}^{\infty}\left|F, S_{t} G\right\rangle \mid d t\right]^{2(n-m)}
$$

Proof. From (3.3) it follows that the modulus of the expression (3.1) is majorized by

$$
\begin{aligned}
2^{n} \sum_{m=0}^{n-1} \sum_{2 \leqq q_{1}<\ldots<q_{m}} \leqq n & \left.\sum_{1 \leqq p_{1}<\ldots<p_{m} \leqq m-1} \sum_{\sigma \in \mathscr{S}_{m}{ }_{m}}\left[\max _{F=f, f^{\prime} ; G=g_{0}, g_{1}} \int_{-\infty}^{\infty} K F, S_{t} G\right\rangle \mid d t\right]^{2(n-m)} \\
& \times z^{2 n-2 m} \int_{0}^{t / z^{2}} d t_{1} \int_{0}^{t_{1}} d t_{2} \ldots \int_{0}^{t_{n-1}} d t_{n} \prod_{h=1}^{m} F\left(t_{q_{h}}-t_{p_{h}}\right)
\end{aligned}
$$

where $2^{n}$-factor comes from summation over all $\varepsilon \in\{0,1\}^{n}$ and

$$
F(u):=\max _{\varepsilon \in\{0,1\}}\left|\left\langle g_{\varepsilon}, S_{t} g_{\varepsilon}\right\rangle\right|
$$

By Theorem (4.1), the expression (4.5) is majorized by

$$
\begin{aligned}
\sum_{k=0}^{n} & \sum_{1 \leqq i_{1}<\ldots<i_{k} \leqq n} \sum_{m=0}^{n-1}\left(\begin{array}{c}
n \\
m
\end{array}\right)^{2} \cdot\left[\max _{F=f, f^{\prime} ; G=g_{0}, g_{1}} \int_{-\infty}^{\infty}\left|\left\langle F, S_{t} G\right\rangle\right| d t\right]^{2(n-m)} \\
& \times \max _{m \leqq n_{0} \leqq n} \frac{t^{n-m}}{\left(n-n_{0}\right) !\left(n_{0}-m\right) !} \cdot\left(\left\|g_{0}\right\|_{-} \vee\left\|g_{1}\right\|_{-}\right)^{2 m} \\
\leqq & 16^{n} \max _{0 \leqq m \leqq n-1}\left(\frac{t^{n-m}}{(n-m) !} \cdot\left(\left\|g_{0}\right\|_{-} \vee\left\|g_{1}\right\|_{-}\right)^{2 m}\right. \\
& \left.\times\left[\max _{F=f, f^{\prime} ; G=g_{0}, g_{1}} \int_{-\infty}^{\infty}\left|\left\langle F, S_{t} G\right\rangle\right| d t\right]^{2(n-m)}\right),
\end{aligned}
$$

which easily implies (4.4).

Corollary (4.3). For each $D \in B\left(H_{0}\right)$ if

$$
\left(\left\|g_{0}\right\|_{-} \vee\left\|g_{1}\right\|_{-}\right)^{2}<\frac{1}{16\|D\|}
$$

then the series obtained replacing, in the matrix element (1.23), $U_{t / z^{2}}$ by its iterated series expansion converges absolutely and uniformly for $z>0$.

Proof. It is enough to notice that this series is nothing but the sum, over all natural integers $n$ and over all $\varepsilon \in\{0,1\}^{n}$ of the terms $\Delta_{n}^{\varepsilon}(z, t)$, defined by (3.3).

\section{The Low Density Limit and Its Derivative}

In this section, using the results of Sects. 3 and 4 we derive the existence of the low density limit (1.23) and its explicit form. Using the latter, we deduce an integral equation for this limit.

Theorem (5.1). For each $u, v \in H_{0}$ and $D \in B\left(H_{0}\right)$ satisfying (1.11) and for each $g_{0}, g_{1} \in K$ satisfying (1.12) and (4.8), the low density limit (1.23) exists and is equal to

$$
\sum_{n=0}^{\infty} \sum_{\varepsilon \in\{0,1\}^{n}}\left\langle u, D_{\varepsilon(1)} \ldots D_{\varepsilon(n)} v\right\rangle \cdot I_{n}^{\varepsilon}(t)
$$

where $I_{n}^{\varepsilon}(t)$ is defined by (3.21). 
Proof. Expanding $U_{t / z^{2}}$ in (1.23) by the iterated series, we obtain

$$
\sum_{n=0}^{\infty} \sum_{\varepsilon \in\{0,1\}^{n}}\left\langle u, D_{\varepsilon(1)} \ldots D_{\varepsilon(n)} v\right\rangle \cdot\left(I_{n}^{\varepsilon}(z, t)+I I_{n}^{\varepsilon}(z, t)\right)
$$

Because of the uniform estimate one has

$$
\begin{gathered}
\lim _{z \rightarrow 0}\left\langle u \otimes W\left(z \int_{S / z^{2}}^{T / z^{2}} S_{u} f d u\right) \Phi, U_{t / z^{2}} v \otimes W\left(z \int_{S^{\prime} / z^{2}}^{T^{\prime} / z^{2}} S_{u} f^{\prime} d u\right) \Phi\right\rangle \\
=\sum_{n=0}^{\infty} \sum_{k=0}^{n} \sum_{1 \leqq i_{1}<\ldots<i_{k} \leqq n}\left\langle u, D_{n}\left(i_{1}, \ldots, i_{k}\right) u\right\rangle \lim _{z \rightarrow 0}\left(I_{n}^{\varepsilon}(z, t)+I I_{n}^{\varepsilon}(z, t)\right),
\end{gathered}
$$

and the result follows from Lemma (3.3) and Lemma (3.4).

In order to find an integral equation satisfied by the limit (5.1), we proceed in analogy with the similar problem dealt with in [3].

The basic idea of the procedure goes in several steps:

(i) One writes the matrix element (3.1) as the integral of its derivative (in $t$ ).

(ii) From the step (i) a term $V(s) U_{s / z^{2}}$ arises and, using the form (1.16) of the interaction, one lets the creators act on the coherent vectors.

(iii) In order to obtain an (ordinary) differential equation for the original matrix element, one has to make the annihilators act on the coherent vectors. This introduces a commutator between the annihilators in the interaction and $U_{s / z^{2}}$. (iv) In order to control the behavior of this commutator, one has to expand $U_{s / z^{2}}$ using the iterated series. This control is achieved by using the uniform estimate and variations of the arguments introduced to control the limit itself.

(v) The result of the limiting procedure of point (iv) is not yet the equation looked for, but an auxiliary one, by solving which [cf. Lemmata (5.8), (5.9)] the explicit form of the equation is eventually deduced.

In the following we discuss in more detail the various steps of procedure outlined above. However, we do not reproduce the length conculations because they are based on essentially the same ideas and techniques already introduced in the first part of the paper.

If, after step (i) above one expands $U_{s / z^{2}}$ using the iterated series, the $n^{\text {th }}$ term of this expansion [by using the explicit form (1.16) of $V(t)$ ] will be:

$$
\begin{aligned}
\frac{1}{z^{2}} & -i)^{n-1} \int_{0}^{t} d t_{1} \int_{0}^{t_{1} / z^{2}} d t_{2} \int_{0}^{t_{2}} d t_{3} \ldots \int_{0}^{t_{n}-1} d t_{n} \\
& \times\left\langle u \otimes W\left(z \int_{S / z^{2}}^{T / z^{2}} S_{u} f d u\right) \Phi,\left(D \otimes A^{+}\left(S_{t_{1} / z^{2}} g_{0}\right) A\left(S_{t_{1} / z^{2}} g_{1}\right)\right.\right. \\
& \left.\left.-D^{+} \otimes A^{+}\left(S_{t_{1} / z^{2}} g_{1}\right) A\left(S_{t_{1} / z^{2}} g_{0}\right)\right) \cdot V\left(t_{2}\right) \ldots V\left(t_{n}\right) v \otimes W\left(z \int_{S^{\prime} / z^{2}}^{T^{\prime} / z^{2}} S_{u} f^{\prime} d u\right) \Phi\right\rangle .
\end{aligned}
$$

Now we write (5.4) as the sum of four terms

$$
I_{n}(1, z, t)+I_{n}(2, z, t)+I I_{n}(0, z, t)+I_{n}(1, z, t)
$$

in two of which $\left(I_{n}(1, z, t), I_{n}(2, z, t)\right)$ the $A, A^{+}$have acted on the coherent vectors giving rise to scalar products; while the remaining two $\left(I I_{n}(0, z, t), I I_{n}(1, z, t)\right)$ 
contain the commutators needed to produce these scalar products. Explicitly,

$$
\begin{aligned}
& I_{n}(1, z, t) \\
& =\int_{0}^{t} d t_{1} \int_{S / z^{2}}^{T / z^{2}}\left\langle S_{u} f, S_{t_{1} / z^{2}} g_{0}\right\rangle d u \cdot \int_{S^{\prime} / z^{2}}^{T^{\prime} / z^{2}}\left\langle S_{t_{1} / z^{2}} g_{1}, S_{u} f^{\prime}\right\rangle d u \\
& \quad \times(-i)^{n-1} \int_{0}^{t_{1} / z^{2}} d t_{2} \int_{0}^{t_{2}} d t_{3} \ldots \int_{0}^{t_{n}-1} d t_{n} \\
& \quad \times\left\langle D^{+} u \otimes W\left(z \int_{S / z^{2}}^{T / z^{2}} S_{u} f d u\right) \Phi \cdot V\left(t_{2}\right) \ldots V\left(t_{n}\right) v \otimes W\left(z \int_{S^{\prime} / z^{2}}^{T^{\prime} / z^{2}} S_{u} f^{\prime} d u\right) \Phi\right\rangle, \\
& I I_{n}(0, z, t)=\int_{0}^{t} d t_{1} \int_{S / z^{2}}^{T / z^{2}}\left\langle S_{u} f, S_{t_{1} / z^{2}} g_{0}\right\rangle d u \cdot(-i)^{n-1} \frac{1}{z} \\
& \quad \times \int_{0}^{t_{1} / z^{2}} d t_{2} \int_{0}^{t_{2}} d t_{3} \ldots \int_{0}^{t_{n-1}} d t_{n}\left\langle D^{+} u \otimes W\left(z \int_{S / z^{2}}^{T / z^{2}} S_{u} f d u\right) \Phi\right. \\
& \left.\quad \times\left[1 \otimes A\left(S_{t_{1} / z^{2}} g_{1}\right), V\left(t_{2}\right) \ldots V\left(t_{n}\right)\right] v \otimes W\left(z \int_{S^{\prime} / z^{2}}^{T^{\prime} / z^{2}} S_{u} f^{\prime} d u\right) \Phi\right\rangle,
\end{aligned}
$$

and $I_{n}(2, z, t)$ [respectively $\left.I_{n}(1, z, t)\right]$ is obtained from $I_{n}(1, z, t)$ [respectively $\left.I I_{n}(0, z, t)\right]$ by: (i) changing the sign; (ii) changing $D$ to $D^{+}$; (iii) exchanging the role of the $g_{0}$ and $g_{1}$ which appear at time $t_{1}$. Now we investigate separately the contributions of the four types of terms (5.5) to the limit (5.1). From Sect. 4.4, we know that

$$
\begin{aligned}
& \mid \int_{0}^{t_{1} / z^{2}} d t_{2} \int_{0}^{t_{2}} d t_{3} \ldots \int_{0}^{t_{n}-1} d t_{n} \\
& \quad \times\left\langle D^{+} u \otimes W\left(z \int_{S / z^{2}}^{T / z^{2}} S_{u} f d u\right) \Phi \cdot V\left(t_{2}\right) \ldots V\left(t_{n}\right) v \otimes W\left(z \int_{S^{\prime} / z^{2}}^{T^{\prime} / z^{2}} S_{u} f^{\prime} d u\right) \Phi\right\rangle \mid \\
& \quad \leqq C(16\|D\|)^{n} \cdot\|u\| \cdot\|v\| \cdot \max _{0 \leqq m \leqq n-1} \frac{t_{1}^{n-m}}{(n-m) !}\left(\left\|g_{0}\right\|_{-} \vee\left\|g_{1}\right\|_{-}\right)^{2 m}
\end{aligned}
$$
with a constant $C$. So, if $\left(\left\|g_{0}\right\|_{-} \vee\left\|g_{1}\right\|_{-}\right)^{2}<\frac{1}{16\|D\|}$, one has, by dominated
convergence,

$$
\begin{aligned}
\lim _{z \rightarrow 0} & \sum_{n=1}^{\infty} I_{n}(1, z, t) \\
= & \sum_{n=1}^{\infty} \lim _{z \rightarrow 0} \int_{0}^{t} d t_{1} \int_{S / z^{2}}^{T / z^{2}}\left\langle S_{u} f, S_{t_{1} / z^{2}} g_{0}\right\rangle d u \cdot \int_{S^{\prime} / z^{2}}^{T^{\prime} / z^{2}}\left\langle S_{t_{1} / z^{2}} g_{1}, S_{u} f^{\prime}\right\rangle d u \\
& \times(-i)^{n-1} \int_{0}^{t_{1} / z^{2}} d t_{2} \int_{0}^{t_{2}} d t_{3} \ldots \int_{0}^{t_{n}-1} d t_{n} \\
& \times\left\langle D^{+} u \otimes W\left(z \int_{S / z^{2}}^{T / z^{2}} S_{u} f d u\right) \Phi \cdot V\left(t_{2}\right) \ldots V\left(t_{n}\right) v \otimes W\left(z \int_{S^{\prime} / z^{2}}^{T^{\prime} / z^{2}} S_{u} f^{\prime} d u\right) \Phi\right\rangle \\
= & \sum_{n=1}^{\infty} \int_{0}^{t} d t_{1} \lim _{z \rightarrow 0} \int_{S / z^{2}}^{T / z^{2}}\left\langle S_{u} f, S_{t_{1} / z^{2}} g_{0}\right\rangle d u \cdot \int_{S^{\prime} / z^{2}}^{T^{\prime} / z^{2}}\left\langle S_{t_{1} / z^{2}} g_{1}, S_{u} f^{\prime}\right\rangle d u \\
& \times(-i)^{n-1} \int_{0}^{t_{1} / z^{2}} d t_{2} \int_{0}^{t_{2}} d t_{3} \ldots \int_{0}^{t_{n-1}} d t_{n} \\
& \times\left\langle D^{+} u \otimes W\left(z \int_{S / z^{2}}^{T / z^{2}} S_{u} f d u\right) \Phi \cdot V\left(t_{2}\right) \ldots V\left(t_{n}\right) v \otimes W\left(z \int_{S^{\prime} / z^{2}}^{T^{\prime} / z^{2}} S_{u} f^{\prime} d u\right) \Phi\right\rangle .
\end{aligned}
$$


Now, if $\left(\left\|g_{0}\right\|_{-} \vee\left\|g_{1}\right\|_{-}\right)^{2}<\frac{1}{16\|D\|}$, then the limit (5.1) is continuous for $u, v \in H_{0}$. So we can write this limit as

$$
\langle u, G(t)\rangle
$$

with $G(t) \in H_{0}$ for each $t \geqq 0$. With this notation (5.7) becomes

$$
\lim _{z \rightarrow 0} \sum_{n=1}^{\infty} I_{n}(1, z, t)=\int_{0}^{t} d t_{1}\left(f \mid g_{0}\right) \chi_{[S, T]}\left(t_{1}\right) \cdot\left(g_{1} \mid f^{\prime}\right) \chi_{\left[S^{\prime}, T^{\prime}\right]}\left(t_{1}\right)\left\langle D^{+} u, G\left(t_{1}\right)\right\rangle
$$

Similarly, we obtain

$$
\lim _{z \rightarrow 0} \sum_{n=1}^{\infty} I_{n}(2, z, t)=\int_{0}^{t} d t_{1}\left(f \mid g_{1}\right) \chi_{[S, T]}\left(t_{1}\right) \cdot\left(g_{0} \mid f^{\prime}\right) \chi_{\left[S^{\prime}, T^{\prime}\right]}\left(t_{1}\right)\left\langle-D u, G\left(t_{1}\right)\right\rangle .
$$

Now, we investigate $I I_{n}(0, z, t)$. By definition for $n \geqq 2$,

$$
\begin{aligned}
I_{n}(0, z, t)= & \int_{0}^{t} d t_{1} \int_{S / z^{2}}^{T / z^{2}}\left\langle S_{u} f, S_{t_{1} / z^{2}} g_{0}\right\rangle d u \cdot(-i)^{n-1} \frac{1}{z} \\
& \times \int_{0}^{t_{1} / z^{2}} d t_{2} \int_{0}^{t_{2}} d t_{3} \ldots \int_{0}^{t_{n-1}} d t_{n}\left\langle D^{+} u \otimes W\left(z \int_{S / z^{2}}^{T / z^{2}} S_{u} f d u\right) \Phi\right. \\
& \left.\times\left[1 \otimes A\left(S_{t_{1} / z^{2}} g_{1}\right), V\left(t_{2}\right) \ldots V\left(t_{n}\right)\right] v \otimes W\left(z \int_{S^{\prime} / z^{2}}^{T^{\prime} / z^{2}} S_{u} f^{\prime} d u\right) \Phi\right\rangle,
\end{aligned}
$$

and we want to consider the limit

$$
\lim _{z \rightarrow 0} I I_{n+1}(0, z, t) \text {. }
$$

Putting $s=t_{1}, s_{1}=t_{2}, \ldots, s_{n}=t_{n+1}$, one finds that

$$
\begin{aligned}
I I_{n+1}(0, z, t)= & \int_{0}^{t} d s \int_{(S-s) / z^{2}}^{(T-s) / z^{2}}\left\langle S_{u} f, g_{0}\right\rangle d u \cdot(-i)^{n} \frac{1}{z} \\
& \times \int_{0}^{s / z^{2}} d s_{1} \int_{0}^{s_{1}} d s_{2} \ldots \int_{0}^{s_{n}-1} d s_{n}\left\langle D^{+} u \otimes W\left(z \int_{S / z^{2}}^{T / z^{2}} S_{u} f d u\right) \Phi\right. \\
& \left.\times\left[1 \otimes A\left(S_{s / z^{2}} g_{1}\right), V\left(s_{1}\right) \ldots V\left(s_{n}\right)\right] v \otimes W\left(z \int_{S^{\prime} / z^{2}}^{T^{\prime} / z^{2}} S_{u} f^{\prime} d u\right) \Phi\right\rangle .
\end{aligned}
$$

By Lemma (3.2) and in the notations of formula (3.11), one has

$\left[1 \otimes A\left(S_{s / z^{2}} g_{1}\right), V\left(s_{1}\right) \ldots V\left(s_{n}\right)\right]=\sum_{\varepsilon \in\{0,1\}^{n}}\left[1 \otimes A\left(S_{s / z^{2}} g_{1}\right), D_{\varepsilon(1)} \ldots D_{\varepsilon(n)} \otimes\left(I_{n}^{\varepsilon}+I I_{n}^{\varepsilon}\right)\right]$.

The main idea of the estimates which follow is that the matrix elements of type $I I$ (cf. Sect. 3) will vanish in the limit $z \rightarrow 0$. In order to control the limit of the remaining ones, we reduce the commutator (5.13) to the normal ordered form in which one can separate the contributions of the type $I$ terms from those of the (irrelevant in the limit) type $I I$ terms. More precisely, one can write

$$
I I_{n+1}(0, z, t):=\sum_{\varepsilon \in\{0,1\}^{n}}\left(\Delta_{1}(n, z, \varepsilon, t)+\Delta_{2}(n, z, \varepsilon, t)\right)
$$


with

$$
\begin{aligned}
& \Delta_{1}(n, z, \varepsilon, t)=\left\langle u, D_{\varepsilon(1)} \ldots D_{\varepsilon(n)} v\right\rangle \\
& \times \sum_{m=0}^{k \wedge(n-k)} \sum_{\substack{\left(q_{0}, \ldots, q_{m}\right) \\
q_{0}=1}}^{\sum^{\prime}} \sum_{m^{\prime}=0}^{k \wedge(n-k)} \sum_{\left(q_{1}^{\prime}, \ldots, q_{m}^{\prime}\right)}^{\prime} \int_{0}^{t} d s \int_{(S-s) / z^{2}}^{(T-s) / z^{2}}\left\langle S_{u} f, g_{0}\right\rangle d u \\
& \times(-i)^{n} \frac{1}{z} \int_{0}^{s / z^{2}} d s_{1} \int_{0}^{s_{1}} d s_{2} \ldots \int_{0}^{s_{n-1}} d s_{n} \\
& \times \prod_{h=0}^{m}\left\langle g_{1}, S_{\left(s_{q_{h}}-s_{q_{h}-1}\right)} g_{1}\right\rangle \cdot \prod_{h=1}^{m^{\prime}}\left\langle g_{0}, S_{\left(s_{q_{h}^{\prime}}-s_{\left.q_{h}^{\prime}-1\right)}\right.} g_{0}\right\rangle \\
& \times\left\langle W\left(z \int_{S / z^{2}}^{T / z^{2}} S_{u} f d u\right) \Phi \cdot \prod_{\alpha \in\{2, \ldots, n\} \backslash\left(\left\{q_{h}\right\}_{h^{m}=1}^{m} \cup\left\{q^{\prime}\right\}_{h}^{\prime} h^{\prime}=1\right)} A^{+}\left(S_{s_{\alpha}} g_{\varepsilon(\alpha)}\right)\right. \\
& \left.\times \prod_{\alpha \in\{1, \ldots, n\} \backslash\left(\left\{q_{h}-1\right\}_{h}^{m}=1 \cup\left\{q_{h}^{\prime}-1\right\}_{h=1}^{m^{\prime}}\right)} A\left(S_{s_{\alpha}} g_{1-\varepsilon(\alpha)}\right) W\left(z \int_{S^{\prime} / z^{2}}^{T^{\prime} / z^{2}} S_{u} f^{\prime} d u\right) \Phi\right\rangle
\end{aligned}
$$

and

$$
\begin{aligned}
& \Delta_{2}(n, z, \varepsilon, t)=\left\langle u, D_{\varepsilon(1)} \ldots D_{\varepsilon(n)} v\right\rangle \\
& \times \sum_{m=0}^{n-1} \sum_{1=q_{0}<q_{1}<\ldots<q_{m} \leqq n} \sum_{\substack{\left(q_{0}, p_{0}, \ldots, q_{m}, p_{m}\right) \\
q_{0}=1, p=0}}^{\sum_{0}} \int_{0}^{t} d s \int_{(s-s) / z^{2}}^{(T-s) / z^{2}}\left\langle S_{u} f, g_{0}\right\rangle d u \\
& \times(-i)^{n} \frac{1}{n} \int_{0}^{s / z^{2}} d s_{1} \int_{0}^{s_{1}} d s_{2} \ldots \int_{0}^{s_{n}-1} d s_{n} \prod_{h=0}^{m}\left\langle S_{s_{p_{h}}} g_{1-\varepsilon\left(p_{h}\right)}, S_{s_{q_{h}}} g_{\varepsilon\left(q_{h}\right)}\right\rangle \\
& \times\left\langle W\left(z \int_{S / z^{2}}^{T / z^{2}} S_{u} f d u\right) \Phi \cdot \prod_{\alpha \in\{1, \ldots, n\} \backslash\left\{q_{h}\right\}_{h}^{m}=0} A^{+}\left(S_{s_{\alpha}} g_{\varepsilon(\alpha)}\right)\right. \\
& \left.\left.\times \prod_{\alpha \in\{1, \ldots, n\} \backslash\left\{p_{h}\right\} h^{m}=1} A\left(S_{s_{\alpha}} g_{1-\varepsilon(\alpha)}\right)\right) W\left(z \int_{S^{\prime} / z^{2}}^{T^{\prime} / z^{2}} S_{u} f^{\prime} d u\right) \Phi\right\rangle .
\end{aligned}
$$

Applying the same arguments used in the proofs of the results in Sects. 3 and 4 to (5.15a) and (5.15b), we obtain the following estimates:

Lemma (5.2). There exists a constant $C_{1}$ such that for each $n \in \mathbf{N}, \varepsilon=0,1$,

$$
\left|I I_{n+1}(\varepsilon, z, t)\right| \leqq(16\|D\|)^{n}\|u\| \cdot\|v\| C_{1} \cdot \max _{0 \leqq m \leqq n} \frac{t^{n+1-m}}{(n+1-m) !}\left(\left\|g_{0}\right\|-\vee\left\|g_{1}\right\|_{-}\right)^{2 m}
$$

Proof. (5.16) is an immediate consequence of the uniform estimate of Theorem (4.2).

Lemma (5.3). For each $n \in \mathbf{N}$,

$$
\lim _{z \rightarrow 0} \Delta_{2}(n, z, \varepsilon, t)=0 .
$$

Proof. The proof is the same as the proof that the terms of type $I I$ tend to zero as $z \rightarrow 0$.

Lemma (5.4). For each $n \in \mathbf{N}$, the limit

exists.

$$
\lim _{z \rightarrow 0} \Delta_{1}(n, z, \varepsilon, t)
$$


Proof. The proof is the same as that used to prove that the terms of type $I$ tend to a limit as $z \rightarrow 0$.

Corollary (5.5). For each $n \in \mathbf{N}, \varepsilon=0,1$ the limit

$$
\lim _{z \rightarrow 0} I I_{n+1}(\varepsilon, z, t)
$$

exists.

Proof. Clear from (5.14) and Lemmata (5.2), (5.3).

Now, for each $n \in \mathbf{N}$, consider the commutator

$$
\begin{aligned}
{[1 \otimes} & \left.A\left(S_{s / z^{2}} g_{1}\right), V\left(s_{1}\right) \ldots V\left(s_{n}\right)\right] \\
= & {\left[1 \otimes A\left(S_{s / z^{2}} g_{1}\right), V\left(s_{1}\right)\right] V\left(s_{2}\right) \ldots V\left(s_{n}\right) } \\
& +\sum_{j=2}^{n} V\left(s_{1}\right) \ldots V\left(s_{j-1}\right)\left[1 \otimes A\left(S_{s / z^{2}} g_{1}\right), V\left(s_{j}\right)\right] V\left(s_{j+1}\right) \ldots V\left(s_{n}\right) .
\end{aligned}
$$

The following lemma proves that the matrix elements of the second term of the right-hand side of (5.20) tend to zero rapidly as $z \rightarrow 0$.

Lemma (5.6). The limit

$$
\begin{aligned}
& \lim _{z \rightarrow 0} \int_{0}^{t} d s \int_{(S-s) / z^{2}}^{(T-s) / z^{2}}\left\langle S_{u} f, g_{0}\right\rangle d u \cdot(-i)^{n} \frac{1}{z} \int_{0}^{s / z^{2}} d s_{1} \int_{0}^{s_{1}} d s_{2} \ldots \int_{0}^{s_{n-1}} d s_{n} \\
& \quad \times\left\langle D^{+} u \otimes W\left(z \int_{s / z^{2}}^{T / z^{2}} S_{u} f d u\right) \Phi \cdot \sum_{j=2}^{n} V\left(s_{1}\right) \ldots V\left(s_{j-1}\right)\right. \\
& \left.\left[1 \otimes A\left(S_{s / z^{2}} g_{1}\right), V\left(s_{j}\right)\right] V\left(s_{j+1}\right) \ldots V\left(s_{n}\right) v \otimes W\left(z \int_{S^{\prime} / z^{2}}^{T^{\prime} / z^{2}} S_{u} f^{\prime} d u\right) \Phi\right\rangle
\end{aligned}
$$

exists and is equal to zero.

Proof.

$$
\begin{aligned}
\int_{0}^{t} d s & \int_{(S-s) / z^{2}}^{(T-s) / z^{2}}\left\langle S_{u} f, g_{0}\right\rangle d u \cdot(-i)^{n} \frac{1}{z} \int_{0}^{s / z^{2}} d s_{1} \int_{0}^{s_{1}} d s_{2} \ldots \int_{0}^{s_{n}-1} d s_{n} \\
& \times\left\langle D^{+} u \otimes W\left(\int_{s^{\prime} z^{2}}^{T / z^{2}} S_{u} f d u\right) \Phi \cdot \sum_{j=2}^{n} V\left(s_{1}\right) \ldots V\left(s_{j-1}\right)\right. \\
& \left.\left.\times\left[1 \otimes A\left(S_{s / z^{2}} g_{1}\right), V\left(s_{j}\right)\right] V s_{j+1}\right) \ldots V\left(s_{n}\right) u \otimes W\left(z \int_{S^{\prime} / z^{2}}^{T^{\prime} / z^{2}} S_{u} f^{\prime} d u\right) \Phi\right\rangle \\
= & z \int_{0}^{t / z^{2}} d s \int_{S / z^{2}-s}^{T / z^{2}-s}\left\langle S_{u} f, g_{0}\right\rangle d u \cdot(-i)^{n} \int_{0}^{s} d s_{1} \int_{0}^{s_{1}} d s_{2} \ldots \int_{0}^{s_{n-1}} d s_{n} \\
& \times\left\langle D^{+} u \otimes W\left(z \int_{s / z^{2}}^{T / z^{2}} S_{u} f d u\right) S_{u} f d u\right) \Phi \cdot \sum_{j=2}^{n} V\left(s_{1}\right) \ldots V\left(s_{j-1}\right) \\
& \left.\times\left[1 \otimes A\left(S_{s} g_{1}\right), V\left(s_{j}\right)\right] V\left(s_{j+1}\right) \ldots V\left(s_{n}\right) v \otimes W\left(z \int_{S^{\prime} / z^{2}}^{T^{\prime} / z^{2}} S_{u} f^{\prime} d u\right) \Phi\right\rangle .
\end{aligned}
$$

Notice that each term of the right-hand side of (5.22) is in $\Delta_{2}(n, z, \varepsilon, t)$, so, by (5.17), one gets (5.21).

Similarly, we get the following 
Lemma (5.7). For each $n \in \mathbf{N}$, the limit

$$
\lim _{z \rightarrow 0} I I_{n+1}(\varepsilon, z, t)
$$

exists and is equal to

$$
\begin{aligned}
\lim _{z \rightarrow 0} & \int_{0}^{t / z^{2}} d s \int_{S / z^{2}-s}^{T / z^{2}-s}\left\langle S_{u} f, g_{\varepsilon}\right\rangle d u \cdot(-i)^{n} \int_{0}^{s} d s_{1} \int_{0}^{s_{1}} d s_{2} \ldots \int_{0}^{s_{n-1}} d s_{n} \\
& \times\left\langle D_{\varepsilon}^{+} u \otimes W\left(z \int_{S / z^{2}}^{T / z^{2}} S_{u} f d u\right) \Phi \cdot\left[1 \otimes A\left(S_{s} g_{1-\varepsilon}\right), V\left(s_{1}\right)\right]\right. \\
& \left.\times V\left(s_{2}\right) \ldots V\left(s_{n}\right) v \otimes W\left(z \int_{S^{\prime} / z^{2}}^{T^{\prime} / z^{2}} S_{u} f^{\prime} d u\right) \Phi\right\rangle .
\end{aligned}
$$

Let us introduce, for simplicity the notation

$$
\Phi_{z}(f, S, T):=W\left(z \int_{S / z^{2}}^{T / z^{2}} S_{u} f d u\right) \Phi .
$$

Then, using Lemma (5.6) and the uniform estimate of Lemma (5.2) we obtain that the limit

$$
\lim _{z \rightarrow 0} \sum_{n=0}^{\infty} I I_{n+1}(\varepsilon, z, t)
$$

exists and is equal to

$$
\begin{aligned}
& \lim _{z \rightarrow 0} z \int_{0}^{t / z^{2}} d s \int_{S / z^{2}-s}^{T / z^{2}-s}\left\langle S_{u} f, g_{\varepsilon}\right\rangle d u \cdot \sum_{n=1}^{\infty}(-i)^{n} \int_{0}^{s} d s_{1} \int_{0}^{s_{1}} d s_{2} \ldots \int_{0}^{s_{n-1}} d s_{n} \\
& \quad \times\left\langle D_{\varepsilon}^{+} u \otimes \Phi_{z}(f, S, T),\left[1 \otimes A\left(S_{s} g_{1-\varepsilon}\right), V\left(s_{1}\right) \ldots V\left(s_{n}\right)\right] v \otimes \Phi_{z}\left(f^{\prime}, S^{\prime}, T^{\prime}\right)\right\rangle .
\end{aligned}
$$

Summing the iterated series inside the commutator and with the change of variables $s z^{2}=r$, we find that the limit (5.26) exists and is equal to

$$
\begin{aligned}
& \lim _{z \rightarrow 0} \int_{0}^{t} d r \chi_{[S, T]}(r)\left(f \mid g_{\varepsilon}\right) d u \\
& \quad \times\left\langle D_{\varepsilon}^{+} u \otimes \Phi_{z}(f, S, T) \frac{1}{z}\left[1 \otimes A\left(S_{r / z^{2}} g_{1-\varepsilon}\right), U_{r / z^{2}}\right] v \otimes \Phi_{z}\left(f^{\prime}, S^{\prime}, T^{\prime}\right)\right\rangle .
\end{aligned}
$$

Clearly, this limit is continuous in $u$ (and in $D_{\varepsilon}^{+} u$ ), hence it will have the form

$$
\left\langle D_{\varepsilon}^{+} u, K_{\varepsilon}(t)\right\rangle=\left\langle u, D_{\varepsilon} K_{\varepsilon}(t)\right\rangle
$$

for some $K_{\varepsilon}(t) \in H_{0}$. Our next step shall be to deduce an equation for $D_{\varepsilon} K_{\varepsilon}(t)$.

Lemma (5.8). Let $K_{\varepsilon}(t)$ and $G(t)$ be defined respectively by Eqs. (5.27), (5.28), and (5.8a). Then $K_{\varepsilon}(t)$ satisfies the equation

$$
\begin{aligned}
\int_{0}^{t}\langle & \left.D_{\varepsilon}^{+} u, K_{\varepsilon}(s)\right\rangle\left(f \mid g_{\varepsilon}\right) \chi_{[s, T]}(s) d s \\
= & \int_{0}^{t} d s\left(f \mid g_{\varepsilon}\right) \chi_{[s, T]}(s)\left(g_{1-\varepsilon} \mid g_{1-\varepsilon}\right)_{-}\left\langle D_{1-\varepsilon}^{+} D_{\varepsilon}^{+} u, K_{1-\varepsilon}(s)\right\rangle \\
& \quad+\int_{0}^{t} d s\left(f \mid g_{\varepsilon}\right) \chi_{[S, T]}(s)\left(g_{1-\varepsilon} \mid g_{1-\varepsilon}\right)_{-} \chi_{\left[S^{\prime}, T^{\prime}\right](s)}\left(g_{\varepsilon} \mid f^{\prime}\right)\left\langle D_{1-\varepsilon}^{+} D_{\varepsilon}^{+} u, G(s)\right\rangle .
\end{aligned}
$$


Proof. The left-hand side of (5.29) can be written, using dominated convergence, (5.27) and Lemma (5.4), as:

Now notice that

$$
\begin{aligned}
\lim _{z \rightarrow 0} & \int_{0}^{t} d s\left(f \mid g_{\varepsilon}\right) \chi_{[S, T]}(s) d s \sum_{n=1}^{\infty}(-i)^{n} \int_{0}^{s / z^{2}} d s_{1} \ldots \int_{0}^{s_{n-1}} d s_{n} \\
& \times\left\langle D_{\varepsilon}^{+} u \otimes \Phi_{z}(f, S, T), \frac{1}{z}\left[1 \otimes A\left(S_{s / z^{2}} g_{1-\varepsilon}\right), V\left(s_{1}\right)\right]\right. \\
& \left.\times V\left(s_{2}\right) \ldots V\left(s_{n}\right) v \otimes \Phi_{z}\left(f^{\prime}, S^{\prime}, T^{\prime}\right)\right\rangle .
\end{aligned}
$$

$$
\left[1 \otimes A\left(S_{s / z^{2}} g_{1-\varepsilon}\right), V\left(s_{1}\right)\right]=i\left(D_{1-\varepsilon} \otimes 1\right)\left\langle S_{s / z^{2}} g_{1-\varepsilon}, S_{s_{1}} g_{1-\varepsilon}\right\rangle A\left(S_{s_{1}} g_{\varepsilon}\right),
$$

therefore, bringing $A\left(S_{s_{1}} g_{\varepsilon}\right)$ to the right of the product $V\left(s_{2}\right) \cdot \ldots \cdot V\left(s_{n}\right)$, we obtain a commutator term plus a term coming from the action of $A\left(S_{s_{1}} g_{\varepsilon}\right)$ on the coherent vector $\Phi_{z}\left(f^{\prime}, S^{\prime}, T^{\prime}\right)$. Thus the limit (5.30) is equal to

$$
\begin{aligned}
\lim _{z \rightarrow 0} & \int_{0}^{t} d s\left(f \mid g_{\varepsilon}\right) \chi_{[S, T]}(s) \sum_{n=1}^{\infty}(-i)^{n-1} \int_{0}^{s / z^{2}} d s_{1} \int_{0}^{s_{1}} d s_{2} \ldots \int_{0}^{s_{n-1}} d s_{n} \\
& \times\left(\left\langleD_{1-\varepsilon}^{+} D_{\varepsilon}^{+} u \otimes \Phi_{z}(f, S, T), \frac{1}{z}\left[1 \otimes A\left(S_{s_{1}} g_{\varepsilon}\right), V\left(s_{2}\right)\right]\right.\right. \\
& \left.\times V\left(s_{3}\right) \ldots V\left(s_{n}\right) v \otimes \Phi_{z}\left(f^{\prime}, S^{\prime}, T^{\prime}\right)\right\rangle \\
& \times\left\langle S_{s / z^{2}} g_{1-\varepsilon}, S_{s_{1}} g_{1-\varepsilon}\right\rangle \\
& +\left\langle D_{1-\varepsilon}^{+} D_{\varepsilon}^{+} u \otimes \Phi_{z}(f, S, T), V\left(s_{2}\right) \ldots V\left(s_{n}\right) v \otimes \Phi_{z}\left(f^{\prime}, S^{\prime}, T^{\prime}\right)\right\rangle \\
& \left.\times \int_{S^{\prime} / z^{2}}^{T^{\prime} / z^{2}} d u\left\langle S_{1} g_{\varepsilon}, S_{u} f^{\prime}\right\rangle d u\right)
\end{aligned}
$$

with the change of variable in the first term:

$$
s_{1} z^{2}=r_{1}
$$

we see that the limit (5.31) is equal to

$$
\begin{aligned}
\lim _{z \rightarrow 0} & \int_{0}^{t} d s\left(f \mid g_{\varepsilon}\right) \chi_{[S, T]}(s) \sum_{n=1}^{\infty}(-i)^{n-1} \int_{0}^{s} d r_{1} \int_{0}^{r_{1} / z^{2}} d s_{2} \int_{0}^{s_{s}} d s_{3} \ldots \int_{0}^{s_{n-1}} d s_{n} \\
& \times\left(\frac { 1 } { z ^ { 2 } } \langle g _ { 1 - \varepsilon } , S _ { ( r _ { 1 } - s ) / z ^ { 2 } } g _ { 1 - \varepsilon } \rangle \cdot \left\langleD_{1-\varepsilon}^{+} D_{\varepsilon}^{+} u \otimes \Phi_{z}(f, S, T),\right.\right. \\
& \left.\frac{1}{z}\left[1 \otimes A\left(S_{r_{1} / z^{2}} g_{\varepsilon}\right), V\left(s_{2}\right)\right] V\left(s_{3}\right) \ldots V\left(s_{n}\right) \cdot v \otimes \Phi_{z}\left(f^{\prime}, S^{\prime}, T^{\prime}\right)\right\rangle \\
& +\left\langle D_{1-\varepsilon}^{+} D_{\varepsilon}^{+} u \otimes \Phi_{z}(f, S, T), V\left(s_{2}\right) \ldots V\left(s_{n}\right) v \otimes \Phi_{z}\left(f^{\prime}, S^{\prime}, T^{\prime}\right)\right\rangle \\
& \left.\times \frac{1}{z^{2}}\left\langle g_{1-\varepsilon}, S_{\left(r_{1}-s\right) / z^{2}} g_{1-\varepsilon}\right\rangle \int_{\left(S^{\prime}-r_{1}\right) / z^{2}}^{\left(T^{\prime}-r_{1}\right) / z^{2}} d v\left\langle g_{\varepsilon}, S_{v} f^{\prime}\right\rangle\right) .
\end{aligned}
$$

The limit (5.33) is the sum of two terms. Concerning the first one, we notice that, because of uniform convergence we can exchange the series with the $d r_{1}$ integral 
and, with the change of variables we obtain the integral

$$
\begin{aligned}
\int_{0}^{t} d s\left(f \mid g_{\varepsilon}\right) \chi_{[S, T]}(s) & \int_{-s z^{2}}^{0} d s_{1}\left\langle g_{1-\varepsilon}, S_{s_{1}} g_{1-\varepsilon}\right\rangle \\
& \times \sum_{n=1}^{\infty}(-i)^{n-1} \int_{0}^{s_{1}+s / z^{2}} d s_{2} \int_{0}^{s_{2}} d s_{3} \ldots \int_{0}^{s_{n-1}} d s_{n} \\
& \times\left\langle D_{1-\varepsilon}^{+} D_{\varepsilon}^{+} n \otimes \Phi_{z}(f, S, T), \frac{1}{z}\left[1 \otimes A\left(S_{s_{1}+s / z^{2}} g_{\varepsilon}\right), V\left(s_{2}\right)\right]\right. \\
& \left.\times V\left(s_{3}\right) \ldots V\left(s_{2}\right) v \otimes \Phi_{z}\left(f^{\prime}, S^{\prime}, T^{\prime}\right)\right\rangle .
\end{aligned}
$$

From Lemma (6.3) of [3] one immediately deduces that, as $z \rightarrow 0$, the limit of the expression (5.34) is the same as

$$
\begin{aligned}
\lim _{z \rightarrow 0} & \int_{0}^{t} d s\left(f \mid g_{\varepsilon}\right) \chi_{[S, T]}(s)\left(g_{1-\varepsilon} \mid g_{1-\varepsilon}\right)_{-} \\
& \times \sum_{n=1}^{\infty}(-i)^{n-1} \int_{0}^{s / z^{2}} d s_{1} \int_{0}^{s_{1}} d s_{2} \ldots \int_{0}^{s_{n-2}} d s_{n-1} \\
& \times\left\langle D_{1-\varepsilon}^{+} D_{\varepsilon}^{+} u \otimes \Phi_{z}(f, S, T), \frac{1}{z}\left[1 \otimes A\left(S_{s / z^{2}} g_{\varepsilon}\right), V\left(s_{1}\right)\right]\right. \\
& \left.\times V\left(s_{2}\right) \ldots V\left(s_{n-1}\right) v \otimes \Phi_{z}\left(f^{\prime}, S^{\prime}, T^{\prime}\right)\right\rangle .
\end{aligned}
$$

By Lemma (5.6), the limit (5.35) is equal to

$$
\begin{aligned}
\lim _{z \rightarrow 0} & \int_{0}^{t} d s\left(f \mid g_{\varepsilon}\right) \chi_{[S, T]}(s)\left(g_{1-\varepsilon} \mid g_{1-\varepsilon}\right)_{-} \\
& \times \sum_{n=1}^{\infty}(-i)^{n-1} \int_{0}^{s / z^{2}} d s_{1} \int_{0}^{s_{1}} d s_{2} \ldots \int_{0}^{s_{n-2}} d s_{n-1} \\
& \times\left\langle D_{1-\varepsilon}^{+} D_{\varepsilon}^{+} u \otimes \Phi_{z}(f, S, T), \frac{1}{z}\left[1 \otimes A\left(S_{s / z^{2}} g_{\varepsilon}\right), V\left(s_{1}\right) V\left(s_{2}\right) \ldots V\left(s_{n-1}\right)\right] v\right. \\
& \left.\otimes \Phi_{z}\left(f^{\prime}, S^{\prime}, T^{\prime}\right)\right\rangle .
\end{aligned}
$$

Thus we can resume the iterated series inside the commutator obtaining that the limit of the expression (5.36) is equal to

$$
\begin{aligned}
\int_{0}^{t} d s( & \left.f \mid g_{\varepsilon}\right) \chi_{[S, T]}(s)\left(g_{1-\varepsilon} \mid g_{1-\varepsilon}\right)_{-} \\
& \quad \times \lim _{z \rightarrow 0}\left\langle D_{1-\varepsilon}^{+} D_{\varepsilon}^{+} u \otimes \Phi_{z}(f, S, T), \frac{1}{z}\left[1 \otimes A\left(S_{s / z^{2}} g_{\varepsilon}\right), U_{s / z^{2}}\right] v \otimes \Phi_{z}\left(f^{\prime}, S^{\prime}, T^{\prime}\right)\right\rangle \\
= & \int_{0}^{t} d s\left(f \mid g_{\varepsilon}\right) \chi_{[S, T]}(s)\left(g_{1-\varepsilon} \mid g_{1-\varepsilon}\right)_{-}\left\langle D_{1-\varepsilon}^{+} D_{\varepsilon}^{+} u, K_{1-\varepsilon}(s)\right\rangle .
\end{aligned}
$$

Concerning the second term we notice that, with the same change of variable (5.32) and again using uniform convergence, it can be written in the form:

$$
\begin{aligned}
\int_{0}^{t} d s\left(f \mid g_{\varepsilon}\right) \chi_{[S, T]}(s) & \int_{-s / z^{2}}^{0} d s_{1}\left\langle g_{1-\varepsilon}, S_{s_{1}} g_{1-\varepsilon}\right\rangle \\
& \times \sum_{n=1}^{\infty}(-i)^{n-1} \int_{0}^{s_{1}+s / z^{2}} d s_{2} \int_{0}^{s_{2}} d s_{3} \ldots \int_{0}^{s_{n-1}} d s_{n} \\
& \times\left\langle D_{1-\varepsilon}^{+} D_{\varepsilon}^{+} u \otimes \Phi_{z}(f, S, T), V\left(s_{2}\right) \ldots V\left(s_{n}\right) v \otimes \Phi_{z}\left(f^{\prime}, S^{\prime}, T^{\prime}\right)\right\rangle \\
& \times \int^{\left(T^{\prime}-z^{2} s_{1}-s\right) / z^{2}} d v\left\langle g_{\varepsilon}, S_{v} f^{\prime}\right\rangle .
\end{aligned}
$$


Again by Lemma (6.3) of [3], the limit of the above expression is equal to

$$
\begin{aligned}
\lim _{z \rightarrow 0} & \int_{0}^{t} d s\left(f \mid g_{\varepsilon}\right) \chi_{[S, T]}(s)\left(g_{1-\varepsilon} \mid g_{1-\varepsilon}\right)_{-} \\
& \times \sum_{n=1}^{\infty}(-i)^{n-1} \int_{0}^{s / z^{2}} d s_{1} \int_{0}^{s_{1}} d s_{2} \ldots \int_{0}^{s_{n-1}} d s_{n} \chi_{\left[S^{\prime}, T^{\prime}\right]}(s)\left(g_{\varepsilon} \mid f^{\prime}\right) \\
& \times\left\langle D_{1-\varepsilon}^{+} D_{\varepsilon}^{+} u \otimes \Phi_{z}(f, S, T), V\left(s_{1}\right) \ldots V\left(s_{n}\right) v \otimes \Phi_{z}\left(f^{\prime}, S^{\prime}, T^{\prime}\right)\right\rangle \\
= & \lim _{z \rightarrow 0} \int_{0}^{t} d s\left(f \mid g_{\varepsilon}\right) \chi_{[S, T]}(s)\left(g_{1-\varepsilon} \mid g_{1-\varepsilon}\right)_{-} \cdot \chi_{\left[S^{\prime}, T^{\prime}\right]}(s)\left(g_{\varepsilon} \mid f^{\prime}\right) \\
& \times\left\langle D_{1-\varepsilon}^{+} D_{\varepsilon}^{+} u \otimes \Phi_{z}(f, S, T), U_{s / z^{2}} v \otimes \Phi_{z}\left(f^{\prime}, S^{\prime}, T^{\prime}\right)\right\rangle
\end{aligned}
$$

and, because of the definition (5.8a) of $G(t)$ and of dominated convergence, this limit is equal to

$$
\int_{0}^{t} d s\left(f \mid g_{\varepsilon}\right) \chi_{[s, T]}(s)\left(g_{1-\varepsilon} \mid g_{1-\varepsilon}\right)_{-} \cdot \chi_{\left[s^{\prime}, T^{\prime}\right]}(s) \cdot\left(g_{\varepsilon} \mid f^{\prime}\right) \cdot\left\langle D_{1-\varepsilon}^{+} D_{\varepsilon}^{+} u, G(s)\right\rangle .
$$

In conclusion from (5.33), (5.37), and (5.39), we obtain Eq. (5.29).

Now, by solving Eq. (5.29), we find the explicit form of $D_{\varepsilon} K_{\varepsilon}(t)$.

Lemma (5.9). In the above notations, denoting for each $\varepsilon \in\{0,1\}$,

$$
\begin{aligned}
D_{g}(\varepsilon): & =\left(1-\left(g_{\varepsilon} \mid g_{\varepsilon}\right)_{-}\left(g_{1-\varepsilon} \mid g_{1-\varepsilon}\right)_{-} D_{\varepsilon} D_{1-\varepsilon}\right)^{-1} \\
& =\sum_{n=0}^{\infty}\left(g_{\varepsilon} \mid g_{\varepsilon}\right)_{-}^{n}\left(g_{1-\varepsilon} \mid g_{1-\varepsilon}\right)_{-}^{n}\left(D_{\varepsilon} D_{1-\varepsilon}\right)^{n},
\end{aligned}
$$

one has

$$
\begin{aligned}
D_{\varepsilon} K_{\varepsilon}(t)= & \chi_{\left[S^{\prime}, T^{\prime}\right]}(t)\left(g_{1-\varepsilon} \mid f^{\prime}\right)\left(g_{\varepsilon} \mid g_{\varepsilon}\right)_{-}\left(g_{1-\varepsilon} \mid g_{1-\varepsilon}\right)_{-} D_{g}(\varepsilon) D_{\varepsilon} D_{1-\varepsilon} D_{\varepsilon} G(t) \\
& +\chi_{\left[S^{\prime}, T^{\prime}\right]}(t)\left(g_{\varepsilon} \mid f^{\prime}\right)\left(g_{1-\varepsilon} \mid g_{1-\varepsilon}\right)_{-} D_{g}(\varepsilon) D_{\varepsilon} D_{1-\varepsilon} D_{\varepsilon} G(t) .
\end{aligned}
$$

Proof. First notice that, since $f, S, T, u$ are arbitrary, then Eq. (5.29) is equivalent to

$$
\begin{aligned}
D_{\varepsilon} K_{\varepsilon}(t)= & \left(g_{1-\varepsilon} \mid g_{1-\varepsilon}\right)_{-} D_{\varepsilon} D_{1-\varepsilon} K_{1-\varepsilon}(t) \\
& +\left(g_{1-\varepsilon} \mid g_{1-\varepsilon}\right)-\left(g_{\varepsilon} \mid f^{\prime}\right) \chi_{\left[S^{\prime}, T^{\prime}\right]}(t) D_{\varepsilon} D_{1-\varepsilon} G(t) .
\end{aligned}
$$

Replacing $K_{1-\varepsilon}(t)$ by its expression (5.27) and with the notation

$$
a_{\varepsilon}:=\left(g_{\varepsilon} \mid g_{\varepsilon}\right)_{-},
$$

we obtain

$$
\begin{aligned}
D_{\varepsilon} K_{\varepsilon}(t)= & \left(a_{\varepsilon} a_{1-\varepsilon} D_{\varepsilon} D_{1-\varepsilon}\right) D_{\varepsilon} K_{\varepsilon}(t) \\
& +\left\{\left(g_{1-\varepsilon} \mid f^{\prime}\right) \chi_{\left[S^{\prime}, T^{\prime}\right]}(s)\left(a_{\varepsilon} a_{1-\varepsilon} D_{\varepsilon} D_{1-\varepsilon}\right) D_{\varepsilon} G(t)\right. \\
& \left.\left.+\left(g_{\varepsilon} \mid f^{\prime}\right) \chi_{\left[S^{\prime}, T^{\prime}\right]}(s) a_{1-\varepsilon} D_{\varepsilon} D_{1-\varepsilon}\right) D_{\varepsilon} G(t)\right\} .
\end{aligned}
$$

Thus $D_{\varepsilon} K_{\varepsilon}(t)$ satisfies the operator equation

$$
\left(1-T_{\varepsilon}\right) D_{\varepsilon} K_{\varepsilon}(t)=G_{\varepsilon}(t),
$$

where $G_{\varepsilon}(t)$ is the term in braces in (5.44) and

$$
T_{\varepsilon}:=a_{\varepsilon} a_{1-\varepsilon} D_{\varepsilon} D_{1-\varepsilon} \text {. }
$$


Notice that

$$
\left\|T_{\varepsilon}\right\| \leqq\left\|g_{0}\right\|_{-}^{2} \cdot\left\|g_{1}\right\|_{-}^{2} \cdot\|D\|^{2}
$$

which is less than (not equal to) 1 because of our assumption (4.8). This implies that

$$
D_{\varepsilon} K_{\varepsilon}(t)=\sum_{n=0}^{\infty} T_{\varepsilon}^{n} G_{\varepsilon}(t)
$$

From this and (5.46), (5.41) immediately follows.

Summing up, one can get the following

Theorem (5.10). The low density limit (1.23), i.e. the quantity (5.8a) satisfies the integral equation

$$
\begin{aligned}
\langle u, G(t)\rangle & \langle u, G(0)\rangle+\int_{0}^{t} d s \sum_{\varepsilon \in\{0,1\}}\left(\sum_{n=1}^{\infty}\left(f \mid g_{\varepsilon}\right) \chi_{[S, T]}(s) \cdot\left(g_{\varepsilon} \mid f^{\prime}\right) \chi_{\left[S^{\prime}, T^{\prime}\right]}(s)\right. \\
& \times\left(g_{1-\varepsilon} \mid g_{1-\varepsilon}\right)_{-}^{n} \cdot\left(g_{\varepsilon} \mid g_{\varepsilon}\right)_{-}^{n-1}\left\langle\left(D_{1-\varepsilon}^{+} D_{\varepsilon}^{+}\right)^{n} u, G(s)\right\rangle \\
& +\sum_{n=1}^{\infty}\left(f \mid g_{\varepsilon}\right) \chi_{[S, T]}(s) \cdot\left(g_{1-\varepsilon} \mid f^{\prime}\right) \chi_{\left[S^{\prime}, T^{\prime}\right]}(s) \\
& \left.\times\left(g_{1-\varepsilon} \mid g_{1-\varepsilon}\right)_{-}^{n-1} \cdot\left(g_{\varepsilon} \mid g_{\varepsilon}\right)_{-}^{n-1}\left\langle D_{\varepsilon}^{+}\left(D_{1-\varepsilon}^{+} D_{\varepsilon}^{+}\right)^{n-1} u, G(s)\right\rangle\right) .
\end{aligned}
$$

In the following, we shall use the notations

$$
D_{1}(\varepsilon):=D_{g}(\varepsilon) D_{\varepsilon}
$$

and

$$
D_{2}(\varepsilon):=\left(g_{1-\varepsilon} \mid g_{1-\varepsilon}\right)_{-} \cdot D_{\varepsilon} D_{1-\varepsilon} D_{g}(\varepsilon) .
$$

Then, (5.48) can be written as

$$
\begin{aligned}
\langle u, G(t)\rangle= & \langle u, G(0)\rangle+\int_{0}^{t} d s \sum_{\varepsilon \in\{0,1\}}\left(\left(f \mid g_{\varepsilon}\right) \chi_{[S, T]}(s) \cdot\left(g_{\varepsilon} \mid f^{\prime}\right) \chi_{\left[s^{\prime}, T^{\prime}\right]}(s)\right. \\
& \left\langle D_{2}^{+}(\varepsilon) u, G(s)\right\rangle+\left(f \mid g_{\varepsilon}\right) \chi_{[s, T]}(s) \\
& \left.\times\left(g_{1-\varepsilon} \mid f^{\prime}\right) \chi_{\left[S^{\prime}, T^{\prime}\right]}(s)\left\langle D_{1}^{+}(\varepsilon) u, G(s)\right\rangle\right) .
\end{aligned}
$$

\section{The Quantum Stochastic Differential Equation}

In this section we identify the integral equation (5.51), satisfied by the low density limit (1.23), with the weak form of the quantum stochastic differential equation

$$
U(t)=1+\int_{0}^{t} \sum_{\varepsilon \in\{0,1\}}\left[D_{1}(\varepsilon) \otimes d N_{s}\left(g_{\varepsilon}, g_{1-\varepsilon}\right)+D_{2}(\varepsilon) \otimes d N_{s}\left(g_{\varepsilon}, g_{\varepsilon}\right)\right] U(s)
$$

on $H_{0} \otimes \Gamma\left(L^{2}(\mathbf{R}) \otimes(K,(\cdot \mid \cdot))\right)$, where $N$ is number process and for each $g, g^{\prime} \in K$,

$$
N_{s}\left(g, g^{\prime}\right):=N\left(\chi_{[0, s]} \otimes|g\rangle\left\langle g^{\prime}\right|\right) \text {. }
$$

Throughout the section we shall use freely the notations, definitions, and results of the Hudson-Parthasarathy paper [17], with the only exception that we call 
"number process" the process called "gauge" in [17] and we denote it $N$ rather than $\Lambda$.

Since $D$ is bounded, it follows that the q.s.d.e. (6.1) has a unique solution $U(t)$ which is given by the iterated series (cf. [17]). Moreover, we have the following

Theorem (6.1). The solution of q.s.d.e. (6.1) is unitary.

Proof. Using the Ito table for the number process (cf. [17]), one knows that the unitarity condition is equivalent to the conditions

$$
\begin{aligned}
& D_{2}^{+}(0)+D_{2}(0)+\left(g_{1} \mid g_{1}\right) D_{1}^{+}(1) D_{1}(1)+\left(g_{0} \mid g_{0}\right) D_{2}^{+}(0) D_{2}(0), \\
& D_{2}^{+}(1)+D_{2}(1)+\left(g_{1} \mid g_{1}\right) D_{2}^{+}(1) D_{2}(1)+\left(g_{0} \mid g_{0}\right) D_{1}^{+}(0) D_{1}(0), \\
& D_{1}^{+}(1)+D_{1}(0)+\left(g_{1} \mid g_{1}\right) D_{1}^{+}(1) D_{2}(1)+\left(g_{0} \mid g_{0}\right) D_{2}^{+}(0) D_{1}(0), \\
& D_{1}^{+}(0)+D_{1}(1)+\left(g_{1} \mid g_{1}\right) D_{2}^{+}(1) D_{1}(1)+\left(g_{0} \mid g_{0}\right) D_{1}^{+}(0) D_{2}(0)
\end{aligned}
$$

[coming from $d\left(U^{+}(t) U(t)\right)=0$ ] together with the analogous conditions coming from $d\left(U(t) U^{+}(t)\right)=0$. We shall prove the above conclusions and this will imply the unitarity.

First of all notice that (5.40), (5.49), and (5.50) implies the following algebraic identities:

$$
\begin{array}{ll}
D_{1}(0) D_{1}^{+}(0)=D_{1}^{+}(1) D_{1}(1) ; & D_{2}(0) D_{2}^{+}(0)=D_{1}^{+}(0) D_{1}(0), \\
D_{1}(1) D_{1}^{+}(1)=D_{1}^{+}(0) D_{1}(0) ; & D_{2}(1) D_{2}^{+}(1)=D_{1}^{+}(1) D_{1}(1), \\
D_{1}(0) D_{2}^{+}(1)=D_{1}^{+}(1) D_{2}(1) ; & D_{2}(0) D_{1}^{+}(1)=D_{2}^{+}(0) D_{1}(0), \\
D_{1}(1) D_{2}^{+}(0)=D_{1}^{+}(0) D_{2}(0) ; & D_{2}(1) D_{1}^{+}(0)=D_{2}^{+}(1) D_{1}(1),
\end{array}
$$

we prove only the first identity, the others are similar.

$$
\begin{aligned}
D_{1}(0) D_{1}^{+}(0)= & D_{g}(0) D_{0} \cdot D_{0}^{+} D_{g}^{+}(0) \\
= & \sum_{m, n=0}^{\infty}\left(g_{1} \mid g_{1}\right)_{-}^{n} \cdot\left(g_{0} \mid g_{0}\right)_{-}^{n} \cdot\left(g_{1} \mid g_{1}\right)_{+}^{m} \cdot\left(g_{0} \mid g_{0}\right)_{+}^{m} \\
& \times\left(D_{0} D_{1}\right)^{n} \cdot\left(-D_{0} D_{1}\right) \cdot\left[\left(D_{0} D_{1}\right)^{+}\right]^{m} \\
= & -\sum_{m, n=0}^{\infty}\left(g_{1} \mid g_{1}\right)_{-}^{n} \cdot\left(g_{0} \mid g_{0}\right)_{-}^{n} \cdot\left(g_{1} \mid g_{1}\right)_{+}^{m} \cdot\left(g_{0} \mid g_{0}\right)_{+}^{m} \cdot D_{0} \cdot\left(D_{0} D_{1}\right)^{n+m} \cdot D_{1} \\
= & \sum_{m, n}^{\infty}\left(g_{1} \mid g_{1}\right)_{-}^{n} \cdot\left(g_{0} \mid g_{0}\right)_{-}^{n} \cdot\left(g_{1} \mid g_{1}\right)_{+}^{m} \cdot\left(g_{0} \mid g_{0}\right)_{+}^{m} \cdot\left(D_{1}\right)^{+} \cdot\left(D_{0} D_{1}\right)^{n+m} \cdot D_{1} \\
= & D_{1}^{+}(1) D_{1}(1) .
\end{aligned}
$$

Therefore, if we prove (6.3a, b, c, d) then their analogues follows from (6.4). Now let us show (6.3a). Recall [form (5.43)] that $\left(g_{\varepsilon} \mid g_{\varepsilon}\right)=: a_{\varepsilon}$ hence by (5.40) and (5.50), one has

$$
a_{\varepsilon} D_{2}(\varepsilon)=D_{g}(\varepsilon)-1 \text {. }
$$

If $\left(g_{0} \mid g_{0}\right)_{-}=0$, the left-hand side of $(6.3 \mathrm{a})$ is equal to

$$
\left(g_{1} \mid g_{1}\right)_{+} D_{1}^{+} D_{0}^{+}+\left(g_{1} \mid g_{1}\right)_{-} D_{0} D_{1}+\left(g_{1} \mid g_{1}\right) D_{1}^{+} D_{1} .
$$

Since $D_{0}=D, D_{1}=-D^{+} ; D_{\varepsilon}^{+}=-D_{1-\varepsilon},(6.7)$ becomes

$$
\left(g_{1} \mid g_{1}\right)_{+} D_{0} D_{1}+\left(g_{1} \mid g_{1}\right)_{-} D_{0} D_{1}-\left(g_{1} \mid g_{1}\right) D_{0} D_{1}=0 .
$$


If $\left(g_{0} \mid g_{0}\right)_{-} \neq 0$, the left-hand side of $(6.3 a)$ is equal to

$$
\begin{aligned}
& \frac{1}{\bar{a}_{0}}\left(D_{g}^{+}(0)-1\right)+\frac{1}{a_{0}}\left(D_{g}(0)-1\right)+\left(a_{1}+\bar{a}_{1}\right) D_{1}^{+} D_{g}^{+}(1) D_{g}(1) D_{1} \\
& \quad+\frac{a_{0}+\bar{a}_{0}}{\left|a_{0}\right|^{2}}\left(D_{g}^{+}(0)-1\right)\left(D_{g}(0)-1\right)
\end{aligned}
$$

so it is equal to zero if and only if

$$
\begin{aligned}
& \left(a_{0}+\bar{a}_{0}\right) D_{g}^{+}(0) D_{g}(0)-\left(a_{0} D_{g}(0)+\bar{a}_{0} D_{g}^{+}(0)\right)+\left(a_{1}+\bar{a}_{1}\right)\left|a_{0}\right|^{2} \\
& \quad \times D_{1}^{+} D_{g}^{+}(1) D_{g}(1) D_{1}=0 .
\end{aligned}
$$

Again using (5.40) we have the identity

$$
D_{g}(1) D_{1}=\sum_{n=0}^{\infty} a_{1}^{n} a_{0}^{n}\left(D_{1} D_{0}\right)^{n} D_{1}=\sum_{n=0}^{\infty} a_{1}^{n} a_{0}^{n} D_{1}\left(D_{0} D_{1}\right)^{n}=D_{1} D_{g}(0)
$$

which gives

$$
\begin{aligned}
D_{1}^{+} D_{g}^{+}(1) D_{g}(1) D_{1} & =D_{g}^{+}(0) D_{1}^{+} D_{1} D_{g}(0)=-D_{g}^{+}(0) D_{0} D_{1} D_{g}(0) \\
& =-D_{g}^{+}(0) D_{g}(0) D_{0} D_{1}=D_{g}^{+}(0) D_{g}(0) D D^{+} .
\end{aligned}
$$

Moreover, (5.40) implies that

$$
\begin{aligned}
a_{0} D_{g}(0)+\bar{a}_{0} D_{g}^{+}(0) & =\frac{a_{0}}{1+a_{0} a_{1} D D^{+}}+\frac{\bar{a}_{0}}{1+\bar{a}_{0} \bar{a}_{1} D D^{+}} \\
& =\frac{\left(a_{0}+\bar{a}_{0}\right)+\left|a_{0}\right|^{2}\left(a_{1}+\bar{a}_{1}\right) D D^{+}}{\left(1+a_{0} a_{1} D D^{+}\right)\left(1+\bar{a}_{0} \bar{a}_{1} D D^{+}\right)} \\
& \left.=D_{g}^{+}(0) D_{g}(0)\left(a_{1}+\bar{a}_{1}\right)\left|a_{0}\right|^{2} D D^{+}+a_{0}+\bar{a}_{0}\right) .
\end{aligned}
$$

From (6.12), (6.13) one immediately obtains (6.10). Similarly, one can prove (6.3b, c, d), so $U$ is unitary.

We sum up our results in the following theorem

Theorem (6.2). For each $f, f^{\prime}, g_{0}, g_{1} \in K, u, v \in H_{0}, D \in B\left(H_{0}\right)$ satisfying (1.11), $S, T, S^{\prime}, T^{\prime} \in \mathbf{R}, t \geqq 0$, if $\left\|g_{0}\right\|_{-} \vee\left\|g_{1}\right\|_{-}<\frac{1}{16\|D\|}$ and $\left\langle g_{0}, S_{t} g_{1}\right\rangle=0, \forall t \geqq 0$, then the low density limit

$$
\lim _{z \rightarrow 0}\left\langle u \otimes W\left(z \int_{S / z^{2}}^{T / z^{2}} S_{u} f d u\right) \Phi, U_{t / z^{2}} v \otimes W\left(z \int_{S^{\prime} / z^{2}}^{T^{\prime} / z^{2}} S_{u} f^{\prime} d u\right) \Phi\right\rangle
$$

exists, where

$$
\frac{d}{d t} U_{t}=-i V(t) U_{t}
$$

and

$$
V(t)=i\left(D \otimes A^{+}\left(S_{t} g_{0}\right) A\left(S_{t} g_{1}\right)-D^{+} \otimes A^{+}\left(S_{t} g_{1}\right) A\left(S_{t} g_{0}\right)\right) .
$$

Moreover, the low density limit (6.14) is equal to

$$
\left\langle u \otimes W\left(\chi_{[S, T]} \otimes f\right) \Psi, U(t) v \otimes W\left(\chi_{\left[S^{\prime}, T^{\prime}\right]} \otimes f^{\prime}\right) \Psi\right\rangle,
$$


where $U(t)$ is the solution of q.s.d.e. (6.1) on $H_{0} \otimes \Gamma\left(L^{2}(\mathbf{R}) \otimes(K,(\cdot \mid \cdot))\right)$, where

(i) $\Psi$ is the vacuum of $\Gamma\left(L^{2}(\mathbf{R}) \otimes(K,(\cdot \mid \cdot))\right)$;

(ii) for each $\mathrm{g}, \mathrm{g}^{\prime} \in K, N_{s}\left(\mathrm{~g}, \mathrm{~g}^{\prime}\right)$ is the number process

$$
N_{s}\left(g, g^{\prime}\right):=N\left(\chi_{[0, s]} \otimes|g\rangle\left\langle g^{\prime}\right|\right) ;
$$

(iii) $D_{1}(\varepsilon), D_{2}(\varepsilon)$ are given by (5.49), (5.50), respectively.

Proof. Theorem (5.1) has shown that the low density limit (6.14) exists. Now, we shall prove that it is equal to (6.15). Clearly, (6.15) is continuous in $u, v \in H_{0}$, so, one can write (6.15) into

$$
\langle u, F(t)\rangle,
$$

where $F(t) \in H_{0}$. Hence we have

$$
\langle u, F(0)\rangle=\left\langle u \otimes W\left(\chi_{[S, T]} \otimes f\right) \Psi, v \otimes W\left(\chi_{\left[S^{\prime}, T^{\prime}\right]} \otimes f^{\prime}\right) \psi\right\rangle=\langle u, G(0)\rangle .
$$

Moreover,

$$
\begin{aligned}
\langle u, F(t)\rangle= & \langle u, F(0)\rangle+\int_{0}^{t} \sum_{\varepsilon \in\{0,1\}}\left\langle u \otimes W\left(\chi_{[S, T]} \otimes f\right) \Psi,\right. \\
& {\left[D_{1}(\varepsilon) \otimes d N_{s}\left(g_{\varepsilon}, g_{1-\varepsilon}\right)+D_{2}(\varepsilon) \otimes d N_{s}\left(g_{\varepsilon}, g_{\varepsilon}\right)\right] v } \\
& \left.\otimes W\left(\chi_{\left[S^{\prime}, T^{\prime}\right]} \otimes f^{\prime}\right) \Psi\right\rangle U(s) .
\end{aligned}
$$

Apply Theorem (4.3) of [17] to (6.17), one obtains

$$
\begin{aligned}
\langle u, F(t)\rangle= & \langle u, F(0)\rangle+\int_{0}^{t} d s \sum_{\varepsilon \in\{0,1\}}\left(f \mid g_{\varepsilon}\right) \chi_{[s, T]}(s) \cdot\left(g_{\varepsilon} \mid f^{\prime}\right) \chi_{\left[S^{\prime}, T^{\prime}\right]}(s) \\
& \times\left\langle D_{2}^{+}(\varepsilon) u, F(s)\right\rangle+\left(f \mid g_{\varepsilon}\right) \chi_{[s, T]}(s) \\
& \left.\times\left(g_{1-\varepsilon} \mid f^{\prime}\right) \chi_{\left[s^{\prime}, T^{\prime}\right]}(s)\left\langle D_{1}^{+}(\varepsilon) u, F(s)\right\rangle\right) .
\end{aligned}
$$

Since (6.1) has unique solution, one knows that (6.18) has a unique solution. Therefore,

$$
\langle u, F(t)\rangle=\langle u, G(t)\rangle, \quad t \geqq 0 .
$$

Acknowledgements. It is our pleasure to thank Robert Alicki and Alberto Frigerio for several fruitful and illuminating conversations on the low density limit problem.

\section{References}

1. Accardi, L.: On the quantum Feynman-Kac formula. Rendiconti Mat. Fisico, Milano 48, 135-180 (1978)

2. Accardi, L.: Noise and dissipation in quantum theory, to appear in Rev. Modern Phys. (1991)

3. Accardi, L., Frigerio, A., Lu, Y.G.: The weak coupling limit as a quantum functional central limit. Commun. Math. Phys. 131, 537-570 (1990)

4. Accardi, L., Frigerio, A., Lu, Y.G.: On the weak coupling limit (II): Langevin equation and finite temperature case, submitted to R.I.M.S.

5. Accardi, L., Frigerio, A., Lu, Y.G.: The weak coupling for Fermion case. J. Math. Phys. 32, 1567-1581 (1991)

6. Accardi, L., Lu, Y.G.: On the weak coupling limit for nonlinear interactions. Ascona Stochastic Processes, Physics and Geometry, 1-26 
7. Accardi, L., Lu, Y.G.: On the weak coupling limit without rotating wave approximation. Anna. Ins. J. Poincaré-Theo. Phys. 54(4), pp. 1-24 (1991)

8. Accardi, L., Bach, A.: The harmonic oscillator as quantum central limit of Bernoulli processes. Prob. Theoret. Rel. Fields (to appear)

9. Alicki, R., Frigerio, A.: Quantum Poisson noise and linear Boltzmann equation. Preprint, March 1989

10. Davies, E.B.: Markovian master equation. Commun. Math. Phys. 39, 91-110 (1974)

11. Dümcke, R.: The low density limit for an $N$-level system interacting with a free Bose or Fermi gas. Commun. Math. Phys. 97, 331-359 (1985)

12. Fagnola, F.: A martingale characterization of quantum Poisson process. Preprint February, 1989

13. Frigerio, A.: Quantum Poisson processes: physical motivations and applications. Lecture Notes in Mathematics, vol. 1303, pp. 107-127. Berlin, Heidelberg, New York: Springer 1988

14. Frigerio, A., Maassen, H.: Quantum Poisson processes and dilations of dynamical semigroups. Prob. Theoret. Rel. Fields 83, 489-508 (1989)

15. Grad, H.: Principles of the kinetic theory of gases. Handbuch der Physik, vol. 12. Berlin, Heidelberg, New York: Springer 1958

16. Hudson, R.L., Lindsay, M.: Uses of non-Fock quantum Brownian motion and a quantum martingale representation theorem. Lect. Notes in Math., vol. 1136, pp. 276-305. Berlin, Heidelberg, New York: Springer

17. Hudson, R.L., Parthasarathy, K.R.: Quantum Ito's formula and stochastic evolutions. Commun. Math. Phys. 93, 301-323 (1984)

18. Palmer, P.F.: Ph.D. Thesis, Oxford University

19. Pulé, J.V.: The Bloch equations. Commun. Math. Phys. 38, 241-256 (1974)

20. Kümmerer, B.: Markov Dilation on $W^{*}$-Algebras. J. Funct. Anal. 63, 139-177 (1985)

21. Accardi, L., Alicki, R., Frigerio, A., Lu, Y.G.: An invitation to the weak coupling and low density limits, to appear in: Quantum probability and Application VI

22. Accardi, L., Lu, Y.G.: The low density limit and the quantum Poisson process, to appear in: Proc. $5^{\text {th }}$ Vilnus Conf. Prob. Th.

Communicated by J.L. Lebowitz 
